\title{
Cartografía Temática del Área de Protección de Flora y Fauna Nevado de Toluca y Propuesta de Senderos Geoturísticos ${ }^{\text {th }}$
}

\section{Thematic Cartography of the Flora and Fauna Protection Area of the Nevado de Toluca and geoturistic paths proposal}

Mariana Patricia Jácome-Paz*a ${ }^{*}$ Néstor López Valdés ${ }^{\mathrm{b}}$, Juan Ramón De la Fuente Rivera ${ }^{\mathrm{b}}$, Gloria Itzel Avila Vasquez ${ }^{\mathrm{c}}$, Javier Guzmán-Sánchez ${ }^{\mathrm{d}}$, Ameyalli Rios-Vázquez ${ }^{\mathrm{f}}$, Ramón Espinasa-Pereña ${ }^{\mathrm{e}}$, Elizabeth Rangel Granados ${ }^{\mathrm{b}}$, Alberto Vásquez Serrano ${ }^{\mathrm{b}}$, Rafael Torres-Orozco ${ }^{\mathrm{h}}$, Lizeth Caballero García ${ }^{\mathrm{g}}$, José Luis Arce Saldaña ${ }^{\mathrm{h}}$, Rodrigo Ville-Benavides ${ }^{\mathrm{i}}$, Aurora Cobos Villagrán ${ }^{\mathrm{f}}$, Karina Victoria Peregrino ${ }^{\mathrm{f}}$, Gerardo Damián Rivera García ${ }^{\mathrm{f}}$, Margarita Caballero Miranda ${ }^{\mathrm{a}}$, Francisco Javier Osorno-Covarrubias ${ }^{\mathrm{j}}$

${ }^{a}$ Instituto de Geofísica, UNAM. Circuito de la investigación Científica s/n, Ciudad Universitaria, Delegación Coyoacán, C.P. 04510, Ciudad de México.

${ }^{b}$ Posgrado en Ciencias de la Tierra, UNAM. Unidad de Posgrado, edificio D, primer piso. Cd. Universitaria, Coyoacán, Ciudad de México. ${ }^{c}$ Colegio de Geografía de la Facultad de Filosofía y Letras, UNAM. Circuito Interior. Ciudad Universitaria, s/n. C.P. 04510. México. ${ }^{d}$ Comisión Nacional Forestal, Gerencia Estatal Morelos. 62290, Río Tamazula 30, Vista Hermosa, Cuernavaca, Morelos.

${ }^{e}$ Centro Nacional de Prevención de Desastres, CENAPRED. Av. Delfín Madrigal No. 665, Pedregal de Santo Domingo, Delegación Coyoacán, Ciudad de México. C.P. 04360

${ }^{f}$ Escuela Nacional de Ciencias Biológicas, IPN. Prolongación de Carpio y Plan de Ayala s/n. Col. Santo Tomás CP11340. Del. Miguel Hidalgo. CDMX ${ }^{g}$ Facultad de Ciencias, UNAM. Circuito Exterior s/n, Coyoacán, Cd. Universitaria, C.P. 04510 Ciudad de México.

${ }^{h}$ Instituto de Geología, UNAM. Circuito de la investigación Científica s/n, Ciudad Universitaria, Delegación Coyoacán, C.P. 04510, Ciudad de México ${ }^{i}$ Facultad de Medicina, Circuito Interior, Ciudad Universitaria, Av. Universidad 3000, C.P. 04510

${ }^{j}$ Instituto de Geografía, UNAM. Circuito de la investigación Científica s/n, Ciudad Universitaria, Delegación Coyoacán, C.P. 04510, Ciudad de México.

\section{Resumen}

El Nevado de Toluca es, desde 2013, un Área Natural Protegida en la categoría de Área de Protección de Flora y Fauna de acuerdo con la legislación mexicana. El volcán cuenta con fácil acceso y con una alta afluencia de visitantes, sin embargo, no cuenta con información orientativa y visible in situ, lo cual favorece el deterioro de la zona y las situaciones de riesgo por no conocer las precauciones al adentrarse a una zona de alta y media montaña. Este proyecto presenta una estrategia de fomento a la conservación del entorno natural a través de una serie de mapas que contienen el área cratérica del volcán y sus alrededores, donde se muestra la ubicación de rutas de montaña, bicicleta y senderos interpretativos, así como información contextualizada sobre salud y prevención, además de su geología, historia eruptiva y biodiversidad. Así mismo, se espera que la consulta o uso in situ por parte de los visitantes de la zona, mitigará las situaciones de riesgo y disminuirá los accidentes de montaña provocados por desconocimiento.

Palabras clave: Nevado de Toluca; Riesgo; Ecoturismo; Cartografía; Sendero interpretativo.

\begin{abstract}
The Nevado de Toluca is a Natural Protected Area with the category of Area de Protección de Flora y Fauna, according to the Mexican legislation, since 2013. The volcano can be easily accessed and has a high influx of visitors; however it has no visible guidance material on site, which favors the deterioration of the area and increases the probability of visitors risk situations, due to lack of education about the precautions needed when entering an area of high and medium mountain. This project presents a strategy to promote the conservation of the natural environment through a series of maps containing the crater area of the volcano and its surroundings, showing the location of mountain routes, bicycle and interpretive trails, as well as contextualized information on health and prevention, in addition to its geology, eruptive history and biodiversity. Likewise, it is expected that its consultation or its in situ use by visitors to the area, mitigate the risk situations and reduce mountain accidents caused by ignorance.
\end{abstract}

Keywords: Nevado de Toluca; Risk; Ecotourism; Cartography; Interpretive path. 


\section{Introducción}

La Faja Volcánica Trans-Mexicana (FVTM) es un arco magmático continental, resultado de la subducción de las placas Rivera y Cocos debajo de la placa Norteamericana, constituido por más de 8000 estructuras volcánicas y algunos cuerpos intrusivos, que se extiende por todo el centro de México desde las costas del Pacífico y hasta las costas del Golfo de México (Demant, 1978; Gómez-Tuena et al. 2005; Ferrari et al. 2012). En la FVTM se presenta tanto vulcanismo monogenético como poligenético. El vulcanismo monogenético está asociado a campos volcánicos con conos de escoria y ceniza, así como derrames de lava; mientras que el vulcanismo poligenético está representado por estratovolcanes y estructuras caldéricas.

La FVTM ha sido subdivida tradicionalmente con base en su geología y tectónica en tres sectores: Occidental, Central y Oriental (Demant, 1978; Pasquaré et al., 1987). Estudios recientes retoman la clasificación mencionada y la amplían a cuatro sectores, de tal manera que se incluye al denominado Sector Extremo Oriental (Ferrari et al., 2012).

El Sector Occidental, se encuentra caracterizado por presentar un vulcanismo bimodal, constituido por estratovolcanes y complejos de domos con una composición dacítica-riolítica, así como centros monogenéticos principalmente de composición basáltica; a su vez, el Sector Central se conforma de conos monogenéticos de escoria con flujos de lava asociados, que presentan una composición basáltica-andesítica. A reserva de los volcanes Tancítaro y el Patamban, los estratovolcanes en este sector son inexistentes. Seguidamente, el Sector Oriental exhibe de manera contrastante, un vulcanismo emplazado principalmente en grandes estratovolcanes, calderas y complejos de domos, con una composición andesítica-riolítica, de tal manera que los volcanes monogenéticos de composición basáltica representan solo una pequeña parte del volumen total. Por último, el Sector extremo Oriental está conformado por magmas con firmas alcalinas principalmente, así como una marcada ausencia de estratovolcanes. Cabe destacar que estos sectores se distribuyen con direcciones preferenciales de E-O para la porción Oriental y Central, así como, ONO-ESE para la porción occidental (Demant, 1978; Pasquaré et al., 1987, Carmichael, 2002; GómezTuena et al., 2005; Ferrari et al., 2012; Siebe et al. 2014). El volcán Nevado de Toluca o Chicnauhtécatl (del náhuatl Nueve Colinas; García-Martínez, 2000) se encuentra dentro del sector Oriental de la FVTM (Ferrrari et al. 2012), a 23 km en dirección suroeste de la ciudad de Toluca. Es la cuarta cumbre más alta de México con una altura de $4680 \mathrm{msnm}$, en el Pico del Fraile (Macías, 2005). El volcán presenta dos lagos al interior del cráter, nombrados de El Sol y de La Luna separados por un domo

\footnotetext{
(C) Jácome-Paz M. P. et al. Published by Terra Digitalis. This is an Open Access article distributed under the terms of the Creative Commons Attribution License (https://creativecommons.org/licenses/by-nc-sa/4.0/), which permits non-commencial sharing of the work and adaptions, provided the original work is properly cited and the new creations are licensed under identical terms.
}

*E-mail address: mp. jacomepaz@gmail.com dacítico central conocido como "El Ombligo" (Armienta et al. 2000). Aunque su morfología es cambiante, las áreas aproximadas son: $0.025 \mathrm{~km}^{2}$ para La Luna y $0.175 \mathrm{~km}^{2}$ para El Sol (Caballero-Miranda, 1996). Estos lagos son ecosistemas únicos en México por su aislamiento geográfico y su elevada altitud (4200 msnm). Las condiciones geográficas y climatológicas favorecen que estos lagos tengan características físico-químicas poco comunes para la región tropical, siendo lagos de aguas frías $\left(9.4^{\circ} \mathrm{C}-9.7^{\circ} \mathrm{C}\right)$, con baja precipitación de minerales y con valores de pH 7.2 \pm 0.6 para El Sol y $6.1 \pm 0.5$ para La Luna (Caballero-Miranda, 1996; Alcocer et al., 2004, Dimas-Flores et al. 2008, Szeroczynska et al. 2015). Por su parte, se han encontrado restos de cerámica, barras de copal, púas de maguey y otros objetos depositados como ofrendas por comunidades prehispánicas en el lecho de la Laguna del Sol, indicando que en su momento dichos lagos fueron un importante centro ceremonial (Quezada-Ramírez, 1972; García-Martínez, 2000).

En cuanto al marco legal vigente, el área que alberga al volcán Nevado de Toluca fue decretada en 1936 como Parque Nacional (PN), con cota de 3,000 msnm como límite inferior y el objetivo fundamental de proteger la belleza escénica y la importancia hidrológica. En 1937 la poligonal del PN fue ampliada con el objetivo de constituir una reserva forestal nacional (CONANP, 2016). Sin embargo, debido a la existencia y proliferación de actividades productivas que afectaban parte del área del PN y que exceden la intención de análisis de este trabajo, la categoría de conservación se modificó el $1^{\circ}$ de octubre de 2013 para constituir un Área de Protección de Flora y Fauna (APFF) (DOF, 2013; SEMARNAT, 2016). Uno de los principales objetivos de dicho cambio es la conservación de sus condiciones biológicas y su entorno ecosistémico a través de un aprovechamiento sostenible y una división en polígonos de acuerdo con: cobertura forestal, tipos de vegetación, pendientes, localidades, actividades productivas, zonas arqueológicas, entre otras. El APFF está conformada por un polígono general, con una superficie total de $536 \mathrm{~km}^{2}$, dentro del cual se ubica una zona núcleo denominada "Cráter", con una superficie de $19.4 \mathrm{~km}^{2}$ y una zona de amortiguamiento con una superficie de $516.5 \mathrm{~km}^{2}$ (CONANP, 2016).

Una vez señalado lo anterior, es importante mencionar que además de ser parte del patrimonio natural (geológico, biológico y limnológico), arqueológico y cosmogónico de nuestro país, el APFF es un sistema socioambiental de fácil acceso que promueve el enriquecimiento cultural de la sociedad con base en su entorno.

En la actualidad, el perfil sociocultural de los visitantes que llegan a la zona es por demás amplio, sin embargo, tienen como punto de convergencia, una alta expectativa sobre las posibilidades recreativas y ecoturísticas que esperan encontrar en la zona (Osorio et al. 2011; Osorio et al. 2017), teniendo como principales propósitos, el ascenso a las cumbres (por encima de los $4000 \mathrm{msnm}$ ) y la visita a los lagos que se encuentran dentro del cráter (El Sol y La Luna). Lo anterior genera una densificación sobre la ruta principal de ascenso, que es un camino de terracería que inicia en el poblado de Raíces sobre la 
carretera federal No. 10 La Puerta-Sultepec, Edo. De Mex. y asciende hasta el cráter del volcán, lo cual ocasiona un deterioro de las áreas por donde pasa dicho camino. Aunado a esto, de acuerdo con las encuestas realizadas y reportadas por Osorio et al.(2006, 2011, 2016 y 2017), las expectativas del visitante no siempre son cubiertas debido a la falta de orientación e información in situ sobre el gran patrimonio que alberga el área o debido a su propia imposibilidad física para realizar ciertas actividades por no contar con la preparación o equipo adecuado a priori. Adicionalmente, destacan Osorio et al. (2011), el turismo predominante manifiesta hábitos tradicionales de consumo recreativo (caminata, ciclismo, alpinismo, campismo, cabalgata, comida campestre, fotografía, investigación, otro y paseo en lancha; Osorio et al., 2017) sobre un paisaje natural, más que una auténtica conciencia y compromiso por minimizar el deterioro ambiental que dicha afluencia causa. El visitante hace prevalecer los propósitos recreativos supeditando los conservacionistas por lo que el turismo de naturaleza puede estar muy distante de propiciar un cambio de conducta con respecto a la relación hombre-recursos naturales (e.g. Valle Rodríguez, 2006; Caravilla et al. 2007; Castro Cuellar et al. 2009; Canet et al. 2017).

Es evidente que debido al fácil acceso y la falta de información visible en la zona (prevención, salud, rutas, patrimonio), se promueve el deterioro del APFF, además de generarse situaciones de riesgo para los visitantes, que desconocen cómo conducirse ante eventualidades como el extremo frío de invierno, (de acuerdo con el Servicio Meteorológico Nacional (2010), para la estación Nevado de Toluca, las normales climatológicas entre 1951-2010 indican que las medias normales se encuentran en el rango de $2.3{ }^{\circ} \mathrm{C}-4.3^{\circ} \mathrm{C}$ durante los meses de octubre-marzo; con un mínimo histórico registrado en $2004 \mathrm{de}-10^{\circ} \mathrm{C}$ ), los pequeños deslizamientos de nieve, las intensas tormentas, el mal de montaña, el esfuerzo físico que se requiere en determinados senderos, sólo por mencionar algunas. Los principales accidentes en las montañas mexicanas son atribuibles a la subestimación de los riesgos y a la negligencia (Neyra-Jaúregui, 2012).

La tesis de este trabajo es que la difusión de la información con respecto al entorno natural y la interacción con el mismo aumenta la capacidad de decisión y resiliencia ante dichas eventualidades. Además, se fomenta la conservación del medio ambiente debido a la sensibilización y al entendimiento de los procesos del entorno por parte de la población. Sin embargo, el enfoque de este trabajo, va más allá de comunicar lo mínimo necesario para tales fines, de tal manera que, la información presentada en este proyecto es amplia y diversa con un carácter transdiciplinario, en un lenguaje de fácil aprehensión y con rigor científico, permitiendo generar conciencia, así como un empoderamiento de la población al ofrecer una experiencia cultural enriquecida, desde una perspectiva histórico socioambiental basada en la importancia del patrimonio del APFF Nevado de Toluca. Es importante señalar que este enfoque antagoniza los escasos objetivos, muchas veces de alto riesgo, que los visitantes se plantean como prioritarios al llegar al área; es decir, se puede desviar su atención hacia otros elementos del paisaje y motivar nuevos propósitos, así como distintas actividades a realizar en el lugar.

\section{Metodología y Software}

La metodología planteada para la realización de la cartografía temática tuvo como principal eje comunicar de manera sintética y accesible los distintos aspectos que constituyen al APFF.

Previo a la creación de los mapas, el grupo de colaboradores de este proyecto llevó a cabo una labor de recopilación y análisis de la información existente sobre los diversos contenidos. Los datos geológicos fueron generados y georreferenciados por Torres-Orozco et al. (2017) con datos vectoriales INEGI (2009, 2015) y cartas topográficas escala 1:50,000.

Posteriormente, la información de los mapas se conjuntó, procesó y diseñó a través de la suite del software ArgGis Desktop de la empresa ESRI, en su producto ArcMap versión $10 \circledR$.

Las doce rutas de montaña que se presentan en la cartografía llevan por títulos: Ruta Circunvalación Completa, Ruta Humboldt, Ruta Humboldt-Heilprin ${ }^{1}$, Ruta Laguna de La Luna, Ruta Laguna de El Sol, Ruta Pico del Águila (Segundo pico más alto), Ruta Pico del Fraile (cima), Ruta Circunvalación Humboldt-Laguna de La Luna, Ruta Parque de los VenadosCara sur, Ruta Parque de los Venados-Pico del águila, Ruta Sendero Interpretativo, Rutas Bicicleta (A) y Rutas Bicicleta (B). Las cuales pueden ser visualizadas de manera interactiva en la plataforma en línea de la revista. En el mapa interactivo se puede observar que los polígonos del área protegida abarcan mayor superficie que la utilizada para el diseño de los mapas de cada ruta. La extensión de la cartografía presentada en este trabajo se restringe al edificio del volcán Nevado de Toluca y sus rutas geoturísticas. Los ejes temáticos de la información vertida en la cartografía resultante se describen a continuación.

\subsection{Salud y prevención}

El Nevado de Toluca es considerado, desde el punto de vista médico y la clasificación de Mieske et al. (2010) y Roach et al. (2016), como: Alta montaña cuyos rangos van de 2500 a 3500 msnm y Muy Alta con rangos de 3500 a 5500 msnm, debido a que presenta alturas en la cual es posible desarrollar síntomas de mala salud por la falta de oxígeno. A esta altura existe una disminución de la presión del aire y por lo tanto del oxígeno que llega a los pulmones; el cuerpo sufre cambios adaptativos importantes como incremento de la velocidad del pulso, de las respiraciones, y de la presión arterial, así como cambios de la conducta. Cuando estos cambios son muy intensos y no se pueden regular, es decir, no existe una aclimatación del cuerpo, las personas pueden presentar mal de montaña agudo (dolor de cabeza, náusea, vómito, fatiga, debilidad, mareo y/o trastornos del

\footnotetext{
${ }^{1}$ También conocido como Heilprim y usado indistintamente. Dicho nombre deriva de Angelo Heilprim quien fue presidente de la Sociedad Geográfica de Filadelfia.
} 
sueño) y sus complicaciones: el edema pulmonar y el edema cerebral de altura (Ville-Benavides, 2017; Bärtsh et al. 2013).

En un estudio reciente, Ville-Benavides (2017) reporta que al analizar a 90 visitantes del Nevado de Toluca, $27.6 \%$ presentó mal de montaña agudo, por lo que es un riesgo de salud latente en el área. Las mejores maniobras para prevenir el mal de montaña agudo incluyen una tasa de ascenso lenta que permita la aclimatación del individuo, de no más de $300 \mathrm{~m}$ al día; descender pronto, mantener una hidratación adecuada, mantenerse abrigado y evitar el sobreesfuerzo en la altitud, así como consultar a un médico con experiencia en medicina de altura. Una vez realizado el diagnóstico se debe considerar descender o descansar a la misma altitud y se debe evitar ascender mientras haya síntomas activos. En casos leves se recomienda la ingesta de líquidos, abrigarse, descansar durante un día y administrar medicamentos para el control de los síntomas, tales como anti inflamatorios no esteroideos o antieméticos. Si los síntomas son persistentes o aumentan en su intensidad se debe descender entre 500 y $1000 \mathrm{~m}$, descansar y consultar con un médico o paramédico con experiencia en enfermedades de altura (VilleBenavides, 2017; Johnson y Luks et al. 2016).

En el trabajo cartográfico presentado se dan recomendaciones generales en cada sendero de montaña con el fin de orientar al posible visitante sobre los requerimientos mínimos dentro del APFF.

\subsection{Montañismo (senderos o rutas principales y sus caracte- rísticas)}

Para poder expresar en los mapas la parte fundamental de orientación y guías de senderos, se trabajó con expertos montañistas y ciclistas, tanto independientes como miembros del Club de Exploraciones de México A.C. CEMAC-Toluca. Se realizó una clasificación y una serie de recomendaciones para cada sendero de montaña.

Existen varias clasificaciones o escalas de dificultad de rutas de montaña reconocidas internacionalmente: la clasificación de la Unión Internacional de Asociaciones de Alpinismo (UIAA), que cuenta con 7 grados de dificultad y está representada por números romanos a partir del I (más fácil), hasta el VII (extraordinariamente difícil); la de Estados Unidos con grados de dificultad ascendente entre 5.2 y 5.9; la francesa que va de 1 a 6 en grado de dificultad ascendente, entre otras. En la UIAA, las rutas del Nevado de Toluca caerían en I, II y, alguna excepción, en III (fácil, poco difícil y algo difícil). Neyra-Jáuregui (2012) propuso una clasificación para las montañas mexicanas de acuerdo con su altura, en donde las rutas del Nevado caen en la clasificación de Alta Montaña Baja (4000 msnm-5000 msnm) Intermedia y Fácil (AMBI-AMBF); que corresponden a rutas de menos de $10 \mathrm{~km}$, poca inclinación y aptas para novatos con experiencia en media montaña. Sin embargo, debido a la gran afluencia de visitantes sin experiencia en alta y media montaña, con bajo o nulo conocimiento sobre técnica y prevención en montaña, la clasificación presentada en este trabajo, desarrollada exclusivamente para el Nevado de Toluca, incluye los grados fácil, algo difícil, difícil y muy difícil y se adaptó de acuerdo con: accesibilidad, longitud, tipo de terreno, altura y tiempo estimado de recorrido. Aunado a esto, en el Nevado de Toluca es frecuente la ocurrencia de nieve, por lo que es importante destacar que una ruta sencilla bajo condiciones adversas de clima puede aumentar su grado de dificultad o volverse impracticable (Neyra-Jáuregui, 2012). Algunas de las recomendaciones básicas al visitar una zona con senderos de montaña son contar con: mochila mediana de tirantes y ajuste en la cadera, recipiente de al menos 1 litro de agua y adecuada hidratación, ropa caliente o de abrigo, chaqueta de fibra polar, pantalones de nylon o fibra sintética, rompe vientos, impermeable, gorra, guantes de fibras transpirables, lentes oscuros con protección ultravioleta (UV), bloqueador solar mayor a 30 o 50 FPS, evitar mojarse, botas de media caña, navaja, entre otras (Grieve et al. 2013, NeyraJáuregui, 2012). Según Neyra-Jáuregui (2012), el equipo para alta montaña bajo condiciones de nieve debe ser: crampones, piolet para travesía de glaciar con correa de seguro, botas plásticas o de piel técnicas para nieve o glaciar y casco.

Aunado al nivel de dificultad y la traza de cada ruta, en la cartografía temática de la zona se muestran puntos de referencia populares, así como nombres clásicos de las rutas y de los picos principales (Careaga Pardave, 1988; Neyra-Jáuregui, 2012).

\subsection{Vulcanismo}

El Nevado de Toluca es un estratovolcán que presenta diversos productos asociados a eventos eruptivos que corresponden a distintas dinámicas de emplazamiento, tales como los derrames formados a través de tranquilas emisiones de lava, así como los flujos de bloques y cenizas, resultado de la formación y destrucción de domos, además de los flujos piroclásticos y los depósitos de caída, consecuencia de los grandes eventos explosivos que lograron depositar cenizas a una distancia mayor a $90 \mathrm{~km}$ con respecto al cráter (Bloomfield et al., 1977; García-Palomo et al., 2002; Arce et al., 2003). Los productos más antiguos del volcán tienen una edad de 1.5 Millones de años (Ma) antes del presente (A.P.), y los más jóvenes presentan una edad de 3,300 años A.P. (Cantagrel et al., 1981; Macías et al., 1997; TorresOrozco et al., 2017). Los depósitos se han clasificado tradicionalmente en dos unidades principales: Nevado Viejo y Nevado Reciente (Cantagrel et al., 1981; Torres-Orozco et al., 2017).

\subsubsection{Nevado Viejo}

Esta unidad se ubica temporalmente entre 1.5 y $0.13 \mathrm{Ma}$ A.P. (Pleistoceno-Holoceno) y se encuentra constituida por derrames de composición andesítica-dacítica (57-65\% en peso de $\mathrm{SiO} 2)$, los cuales conforman la estructura principal del edificio, intercalados con productos asociados a episodios explosivos ( 0.9-0.8 Ma A.P.), sin embargo, la actividad efusiva es la que predomina (Cantagrel et al., 1981; García-Palomo et al., 2002; Torres-Orozco et al., 2017). Las lavas presentan plagioclasas, clinopiroxenos, ortopiroxenos y hornblendas, embebidos en matriz vítrea con microfenocristales de plagioclasa. Es común encontrar e identificar fragmentos de estas rocas como accesorios en los depósitos volcanoclásticos y piroclásticos más jóvenes, ya que presentan color de alteración rosado-rojizo 
(García-Palomo et al., 2002). A su vez, debido a la alteración y erosión del volcán, se encuentran depósitos de avalancha, asociados al colapso de flancos (2 colapsos parciales), depósitos de lahar y depósitos fluviales (flujos de lodo), los cuales fueron emplazados principalmente en el sector sur, alcanzando distancias de más de $50 \mathrm{~km}$, rellenando con detritos las depresiones existentes (Cantagrel et al., 1981; Macías et al., 1997; Capra y Macías, 2000; García-Palomo, 2002; Capra et al., 2002); es necesario mencionar que diversos autores reportan un cese en la actividad volcánica para el intervalo de 1.2-0.1 Ma A.P., sin embargo, estudios recientes demuestran que ha existido una actividad continua, contraponiéndose a la idea anterior (TorresOrozco et al., 20017).

\subsubsection{Nevado Reciente}

La unidad moderna del volcán comienza su emplazamiento hace $0.05 \mathrm{Ma}$ y continúa hasta nuestros días, debido a que la última erupción registrada presenta una edad de 3,300 años A.P. (Macías et al., 1997). Los productos son composicionalmente dacíticos (63-67\% en peso de $\mathrm{SiO} 2$ ) y están asociados a una actividad bimodal, con predominancia de la actividad explosiva, con un estilo eruptivo pliniano, de tal manera que la unidad está constituida por el emplazamiento de domos y lavas, así como una compleja secuencia de depósitos como son los piroclásticos de caída, corrientes piroclásticas de densidad, avalanchas de escombros, flujos de bloques y cenizas, además de los depósitos fluviales, flujos de detritos y los propios de la actividad glaciar, estos últimos visibles en los flancos del cráter (Bloomfield and Valastro, 1974, 1977; Bloomfield, 1975; Cantagrel et al., 1981; Macías et al., 1997; Capra and Macías, 2000; Capra et al., 2002). Las lavas presentan un ensamble mineralógico de plagioclasa, ortopiroxeno, hornblenda y biotita.

Durante la formación del Nevado reciente existen al menos 5 episodios de destrucción de grandes domos fechadas en 37,000; 32,000; 28,000; 26,000; y 13,000 años A.P. (Bloomfield et al., 1977; Macías et al., 1997; García-Palomo et al., 2002; Arce et al., 2003; Arce et al., 2005; Capra et al., 2006), así como 5 erupciones plinianas con edades de 42,000; 36,000; 21,700 (Pómez Toluca Inferior, PTI); 12,100 (Pómez Toluca Intermedia, PTIn) y 10,500 (Pómez Toluca Superior, PTS) años A.P. (Bloomfield y Valastro, 1974, 1977; Cantagrel et al., 1981; Heine, 1988; Macías et al., 1997; Newton and Metcalfe, 1999; Caballero et al., 2001; García-Palomo et al., 2002). Es necesario mencionar que el último evento relacionado a la actividad efusiva, corresponde al emplazamiento del domo "El Ombligo", hace 9,100 años A.P. (Arce et al., 2003), mientras que para la actividad explosiva se tiene un depósito de corriente piroclástica de densidad con una edad de 3,300 años A.P. (Macias et al., 1997). De igual manera, se tiene registro de al menos un evento de colapso de la estructura volcánica, para el cual se reporta una edad de 28,000 años A.P. (Caballero, 2007).

Existen otros depósitos que completan la secuencia del Nevado Reciente y son producto de las glaciaciones. Diversos estudios de las montañas de México han demostrado la existencia de varias fases de glaciación en los últimos 200,000 años
(Vázquez Selem y Heine, 2011; White et al., 1990), sin embargo, para el caso del Nevado de Toluca, la intensa actividad volcánica del Cuaternario ha borrado la mayor parte de las evidencias de glaciación, aunque al menos para los últimos 20,000 años, aún se conservan (Heine, 1988). La morfología del Nevado de Toluca presenta evidencia de avance glaciar que fue identificada y clasificada por Vázquez-Selem y Heine (2011): 32,000 años A.P. (MI); 21,700-17,000 años A.P. (MII 1); 17,000-11,600 años A.P. (MII 2); 11,600 años A.P. (late Younger Dryer), 8,000-7,000 años A.P. (MIV) y la pequeña edad de hielo (MV; Siglo XIV-XIX).

\subsection{Biodiversidad}

Gracias a su ubicación en una zona de transición entre las regiones biogeográficas neártica y neotropical, este volcán protege una mezcla diversa de especies (Morrone, 2005). El Nevado de Toluca alberga 1058 especies: 831 especies constituyen la flora y 227 la fauna, 61 de las cuales se encuentran catalogadas en riesgo (NOM-059-SEMARNAT-2010; SEMARNAT, 2016).

Se resalta la existencia de especies de distribución restringida y endémicas de las altas montañas del centro del país: Eryngium monocephalum cav. (conocida como Rosa de montaña), Draba nivicola, Festuca lívida y Trisetum spicatum (conocido como zacate) y especies en alguna categoría de riesgo como el enebro azul (Juniperus sabinoides montícola), la mariposa monarca (Danaus plexippus), tlaconete de Robert (Pseudoeurycea robertsi), rana de árbol plegada o surcada (Hyla plicata), rana de Moctezuma (Lithobates montezumae) y víbora de cascabel (Crotalus transversus) (DOF, 2016; SEMARNAT, 2016).

Además, durante el recorrido de los diversos senderos de montaña, se pueden encontrar cuatro tipos principales de vegetación: bosque de oyamel (Abies religiosa), bosque de ocote (Pinus montezumae), bosque de pino de las alturas (Pinus hartwegii) y en las partes más altas, pastizal alpino (Díaz-Roldán, 2013). Sus bosques son sumamente importantes porque funcionan como zonas de recarga de mantos acuíferos. Gracias a ellos, el agua se infiltra y llega al subsuelo, para después ser aprovechada por la población de Toluca y la Ciudad de México (SEMARNAT, 2016). En cada mapa se mencionan algunos elementos de flora y fauna que el visitante observará en esa ruta, con el fin de enriquecer su experiencia y realzar la gran biodiversidad presente en la zona.

\section{Conclusiones}

La cartografía temática del APFF presentada en este trabajo es un esfuerzo transdisciplinario de alto impacto científico y cultural, con un objetivo claramente social y ambiental con carácter sustentable. El material generado es una importante herramienta visual que puede ser utilizada para realizar consultas académicas, así como material didáctico para la divulgación y el fomento a la conservación de los recursos naturales de la APFF. Adicionalmente, se espera que el uso de este material in situ, así como a través de diversos canales de comunicación, 
convencionales y no convencionales, motive la búsqueda de una experiencia conservacionista por parte de los visitantes del Área Natural Protegida.

Es importante resaltar, que el área es objeto de un programa de gestión y conservación ambiental a través del Plan de Manejo del APFF Nevado de Toluca; sin embargo, éste no contiene un seguimiento de análisis y mitigación de impacto ambiental debido específicamente a la alta afluencia. Además, el análisis del impacto del AFPP no considera la gestión del riesgo como una medida de mitigación de impacto ambiental.

No existe en el área una gestión integral del riesgo que minimice el estado de vulnerabilidad de los visitantes; bajo tal premisa, se sugiere como una importante y primera estrategia de mitigación del riesgo, la implementación "in situ" de la difusión de información y del sendero interpretativo a través de paneles divulgativos con información transdisciplinaria. El objetivo de esta acción sería empoderar directamente al visitante para una mejor toma de decisiones.

\section{Agradecimientos}

A los revisores Dra. Lucia Capra Pedol, Dr. Carles Canet. Al editor Dr. Luca Ferrari, Dra. Penélope López-Quiroz y todo el equipo editorial de Terra Digitalis. A las facilidades otorgadas por la Lic. Sofía Manzur y su equipo de CEPANAF, al Biol. Luis Miguel Mandujano y su equipo de CONANP. A los colegas Maribel Osorio, Denisse Morales Perea, Juan Carlos Gavilanes, Marie Nöelle Guilbaud, Salvador Acosta, Fernando Alberto López Ortiz, Mauricio Alejandro Amaya y Héctor Martínez Campos. A los ciclistas y montañistas Miguel Venegas Orihuela, Iván García Orozco, Jesús Roberto Aguilar Ordoñez, José Silva Sandoval de CEMAC-Toluca y a Daniel Carrillo Cortés. Al autor del video de dron (Jelly Beans Studio, (2017)).

\section{Referencias}

Alcocer, J., Oseguera, L. A., Escobar, E., Peralta, L., Lugo, A., 2004. Phytoplankton biomass and water chemistry in two high mountains, tropical lakes in central Mexico. Arctic, Antarctic and Alpine Research 36, 342-346.

Arce, J. L., Cervantes, K. E., Macías, J. L., Mora, J. C., 2005. The 12.1 ka middle Toluca pumice: a dacitic Plinian-subplian eruption of Nevado de Toluca in Central México. Journal of Volcanology and Geothermal Research 147, 125-143.

Arce, J. L., Macías, J. L., Vazquez, S. L., 2003. The 10.5 ka Plinian eruption of Nevado de Toluca, Mexico: stratigraphy and hazard implications. Geol. Soc. Am. Bull 115 (2), 230-248.

Armienta, M. A., De la Cruz-Reyna, S., Macias, J. L., 2000. Chemical characteristics of the crater lakes of Popocatetetl, El Chichon, and Nevado de Toluca volcanoes. Mexico Journal of Volcanology and Geothermal Research 97, 105-125, doi:10.1016/S0377-0273(99)00157-2.

Barra, F., 2008. Geology of mexico: Celebrating the centenary of the geological society of mexico.: S. a. alaniz-alvarez and a. f. nieto-samaniego, editors. geological society of america, special paper $422,465 \mathrm{pp}$. boulder, colorado. 2007. isbn 13-978-0-8137-2422-5. Economic Geology 103 (3), 653, doi:10.2113/gsecongeo.103.3.653.

Bloomfield, K., 1975. A Late Quaternary monogenetic volcano field in central Mexico. Geologische Rundschau 64, 476-497.

Bloomfield, K., Sánchez-Rubio, G., Wilson, L., 1977. Plinian eruptions of Nevado de Toluca Volcano. Geologische Rundschau 66, 120-146.
Bloomfield, K., Valastro, S., 1974. Late Pleistocene eruptive history of Nevado de Toluca, central Mexico. Geological Society of America Bulletin 85, 901906.

Bloomfield, K., Valastro, S., 1977. Late Quaternary tephrochronology of Nevado de Toluca, central México. Institute of Geological Sciences, Overseas Geology and Mineral Resources 46, 1-15.

Bärtsch, P., Swenson, E. R., 2013. Acute high-altitude illnesses. New England Journal of Medicine 368 (24), 2294-2302, doi:10.1056/NEJMcp1214870.

Caballero, L., 2007. Análisis textural del depósito de avalancha de escombros «El zaguán», volcán Nevado de Toluca: dinámica de transporte y mecanismos de emplazamiento, tesis de maestría. UNAM, México, D. F.

Caballero, M., Macías, J. L., Urrutia-Fucugauchi, J., Lozano-García, S., Castañeda, R., 2001. Volcanic stratigraphy and palaeolimnology of the Upper Lerma Basin during the Late Pleistocene and Holocene. Sedimentology special volume, Lacustrine Volcaniclastic-Sedimentation 30, 57-71.

Caballero, M. E., 1996. The diatom flora of two acid lakes in central Mexico. Diatom Res 11, 227-240.

Cantagrel, J. M., Robin, C. E., Vincent, P., 1981. The main stages of the evolution of an andesitic composite volcano: The nevado de toluca (Mexico). Bull. Volcanol 44, 177e186.

Capra, L., Carreras, L. M., Arce, J. L., Macías, J. L., 2006. The Lower Toluca Pumice: a ca. 21,700 yr B.P. Plinian eruption of Nevado de Toluca volcano, Mexico. Special Paper Geol. Soc. Am 402, 155-173.

Capra, L., Macías, J. L., 2000. Pleistocene cohesive debris flows at Nevado de Toluca Volcano, central Mexico. Journal of Volcanology and Geothermal Research 102, 149-168.

Capra, L., Macías, J. L., 2002. The cohesive Naranjo debris-flow deposit (10 $\mathrm{km} 3$ ): A dam breakout flow derived from the Pleistocene debrisavalanche deposit of Nevado de Colima Volcano (México). Journal of Volcanology and Geothermal Research 117, 213-235.

Carcavilla, L., López-Martínez, J., Durán, J. J., 2007. Patrimonio geológico y geodiversidad: investigación, conservación, gestión y relación con los espacios naturales protegidos. No 7 en Serie de Cuadernos del Museo Geominero. Instituto Geológico y Minero de España, Madrid, 360 pp.

Careaga Pardave, A., 1988. Xinantecatl o Nevado de Toluca. Nevado de Colima. Volcán de Fuego de Colima. Rutas de las Montañas de México. 1975.

Carles, C., Mora-Chaparro, J. M., Iglesias, A., Cruz-Pérez, M. A., SalgadoMartínez, E., Zamudio-Ángeles, D., Fitz-Díaz, E., Martínez-Serrano, G., Gil-Ríos, A., Poch, J., 2017. Cartografía geológica para la gestión del geopatrimonio y la planeación de rutas geoturísticas: Aplicación en el Geoparque Mundial de la UNESCO Comarca Minera, Hidalgo. Terra Digitalis 1 (2), $1-7$.

Carmichael, I., 2002. The andesite aqueduct: perspectives on the evolution of intermediate magmatism in west-central (105-99W) Mexico. Contributions to Mineralogy and Petrology 143, 641-663.

Castro Cuellar, A., Cruz Burguete, J. L., Ruiz-Montoya, L., 2009. Educar con ética y valores ambientales para conservar la naturaleza. Convergencia. Revista de Ciencias sociales 50, UAEM.

CONANP, 2016. Plan de Manejo. apff nevado de toluca, para consulta pública en cumplimiento a lo previsto por los artículos 65 de la ley general del equilibrio ecológico y la protección al ambiente y 73 de su reglamento en materia de áreas naturales protegidas. http://www. conanp.gob.mx/que hacemos/pdf/programas_manejo/RESUMEN_NEVADO_TOLUCA.pdf.

Demant, A., 1978. Características del Eje Neovolcánico Transmexicano y sus problemas de interpretación. Revista Instituto de Geología 2, 172-187.

Dimas Flores, N., Alcocer, J., Ciros Pérez, J., 2007. The structure of the zooplancton assemblages from two neighboring troical high mountain lakes. Journal of Freshwater Ecology 23, 21-31.

DOF, 2010. 30/12/2010 NORMA Oficial Mexicana NOM-059-SEMARNAT2010, Protección ambiental-Especies nativas de México de flora y fauna silvestres-Categorías de riesgo y especificaciones para su inclusión, exclusión o cambio-Lista de especies en riesgo.

DOF, 2013. 01/10/2013DECRETO que reforma, deroga y adiciona diversas disposiciones del diverso publicado el 25 de enero de 1936, por el que se declaró Parque Nacional la montaña denominada "Nevado de Toluca"que fue modificado por el diverso publicado el 19 de febrero de 1937. Plan de Manejo del APFF.

Díaz-Roldán, A., 2013. Diagnosis de la vegetación y Flora del Parque Nacional Nevado de Toluca (Estado de México, México) y propuesta para su gestoría. 
Ph.D. thesis, Facultad de Estudios Superiores Iztacala. Universidad Nacional Autónoma de México, Los Reyes, Iztacala, 71 pp.

Ferrari, L., Orozco-Esquivel, T., Manea, V., Manea, M., 2012. The dynamic history of the trans-mexican volcanic belt and the mexico subduction zone. Tectonophysics 522-523, 122 - 149, doi:10.1016/j.tecto.2011.09.018.

García-Martínez, B., 2000. Los nombres del Nevado de Toluca. Arqueología Mexicana 8 (43), 24-26.

García-Palomo, A., Macias, J. L., Arce, J. L., Capra, L., Garduño, V. H., Espíndola, J. M., 2002. Geology of Nevado de Toluca volcano and surrounding areas, central Mexico. Geol. Soc. Am. Map Ser, 1-48.

Gómez-Tuena, A., Orozco-Esquivel, T., Ferrari, L., 2007. Igneous petrogenesis of the Trans-Mexican Volcanic Belt. In: Alaniz-Álvarez, S.A. Geological Society of America Special Papers, 422, 129-181.

Heine, K., 1988. Late Quaternary glacial chronology of the Mexican volcanoes. Die Geowissenschaften 6, 197-205.

Instituto Nacional de Estadística y Geografía (INEGI), 2009. Conjunto de datos vectoriales de la carta Topográfica Volcán Nevado de Toluca, a escala 1:50 000 (en línea). INEGI, Toluca, México, Información cartográfica y base de datos, acceso libre, consulta: 1 de agosto de 2016, http://www. beta. inegi. org.mx/app/biblioteca/ficha. $\mathrm{html}$ ?upc=702825717797.

Instituto Nacional de Estadística y Geografía (INEGI), 2015. Conjunto de datos vectoriales de la carta Topográfica Volcán Nevado de Toluca, a escala 1:50 00, serie III, (en línea). INEGI, Toluca, México, Información cartográfica y base de datos, acceso libre, consulta: 1 de agosto de 2016, http://www. beta.inegi .org.mx/app/biblioteca/ficha . html?upc $=702825202620$.

Jelly Beans Studio, 2017. El Nevado de Toluca 4K. Publicado el 14 de marzo del 2017 bajo licencia Youtube standar, Consultado el 15 de abril del 2018, Video original: https://www. youtube.com/watch?v=jzMAkLJTPA4\& $\mathrm{t}=44 \mathrm{~s}$.

Johnson, N. J., Luks, A. M., 2016. High-Altitude Medicine. Medical Clinics $100(2), 357-369$.

Macías, J. L., 2005. Geología e historia eruptiva de algunos de los grandes volcanes activos de México.Volumen conmemorativo del Centenario. Temas selectos de la geología mexicana. Boletín de la Sociedad Geológica Mexicana 57 (3), 379-424.

Macías, J. L., Garcia-Palomo, A., Arce, J. L., Siebe, C., Espindola, J. M., Komorowski, J. C., Scott, K. M., Kowallis, B. J., 1997. Late PleistoceneHolocene cataclysmic eruptions at Nevado de Toluca and Jocotitlán volcanoes, Central Mexico. En: BYU Geology Studies. Vol. 42(1). pp. 493-528.

Mieske, K., Flaherty, G., O'Brien, T., 2010. Journeys to High Altitude:Risks and Recommendations for Travelers with Preexisting Medical conditions. Journal of Travel Medicine 17 (1), 48-62.

Morrone, J. J., 2005. Hacia una síntesis biogeográfica de México. Revista Mexicana de Biodiversidad 76 (2), 207-252.

Newton, J. A., Metcalfe, S. E., 1999. Tephrochronololgy of the Toluca Basin, central México. Quaternary Science Reviews 18, 1039-1059.

Neyra Jaúregui, J. A., 2012. Guía de las altas montañas de México y una de Guatemala. Comisión Nacional para el Conocimiento y Uso de la Biodiversidad (CONABIO), México.

Osorio, M., Vallejo, B., Vega, J. L., 2006. Propuesta para el desarrollo turístico sustentable del Área de Protección de Flora y Fauna Nevado de Toluca. Reporte Técnico. Toluca: Universidad Autónoma del Estado de México y Comisión Nacional Forestal.

Osorio, M., Vallejo, B., Vega, J. L., 2016. Plan Estratégico para el Desarro1lo Turístico Sustentable del Parque Nacional Iztaccíhuatl-Popocatépetl y Zoquiapan y su Área de Influencia. Reporte Técnico. Toluca: Universidad Autónoma del Estado de México.

Osorio García, M., Franco Maass, S., Ramírez de la O, I. L., Nava Bernal, G., Novo Espinosa de los Monteros, G., Regil, H. H., 2011. El visitante del Parque Nacional Nevado de Toluca, México. Análisis del comportamiento en un área natural protegida. Investigaciones geográficas (76), 56-70.

Osorio García, M., Monge Amores, L. E., Serrano Barquín, R. C., Cortés Soto, I. Y., 2017. Perfil del visitante de naturaleza en Latinoamérica: prácticas, motivaciones e imaginarios. Estudio comparativo entre México y Ecuador. PASOS. Revista de Turismo y Patrimonio Cultural 15 (3), 713-729.

Pasquarè, G., Vezzoli, L., Zanchi, A., 1987. Morphological and structural model of Mexican Volcanic Belt. Geofísica Internacional 26, 159-176.

Quezada-Ramírez, M. N., 1972. Los matlatzincas, época prehispánica y época

colonial hasta 1650. Instituto Nacional de Antropología e Historia, Departamento de Investigaciones Históricas.

Roach, R. C., Lawley, J., Hackett, P. H., 2016. High Altitude Physiology. En: Auerbach's Wilderness Medicine.

SEMARNAT, 2016. Programa de Manejo Área de Protección de Flora y Fauna Nevado de Toluca. Comisión Nacional de Áreas Naturales Protegidas. México. 317 pp.

Servicio Meteorológico Nacional, S., 2016. Tabla de normales climatólogicas 1951-2010. Consultado en http://smn.cna.gob.mx/es/ informacion-climatologica-ver-estado?estado=mex.

Siebe, C., Guilbaud, M. N., Salinas, S., Kshirsagar, P., Chevrel, O., De la Fuente, J. R., Hernández, J. A., Godínez, L., 2014. Monogenetic vulcanism ff the Michoacan . Guanajuato Volcanic Field: Maar craters of the Zacapu basins and domes, shields, and escorian cones of Tarascan highlands (Paracho Parícutin region). In Pre-meeting field trip (November 13 - 17) Prior to the 5 International Maar Conference (5 IMC - IAVCEI), Querétaro, México. Noviembre.

Szeroczyńska, K., Zawisza, E., Wojewódka, M., 2015. Initial Time Of Two High Altitude Crater Lakes (Nevado De Toluca, Central Mexico) Recorded In Subfossil Cladocera. Studia Quaternaria 32 (2), 109-116, doi:10.1515/squa-2015-0010.

Torres-Orozco, R., Arce, J. L., Layer, P. W., Benowitz, J. A., 2017. The Quaternary history of effusive volcanism of the Nevado de Toluca area, Central Mexico. Journal of South American Earth Sciences 79, 12-39, doi:10.1016/j.jsames.2017.07.008.

Valle Rodríguez, S., 2006. Las Áreas Naturales Protegidas en México. Un ejemplo de propuesta de gestión de una Área Protegida y plan de manejo en La Sierra de Monte Escobedo (Zacatecas, México). Ph.D. thesis, Tesis doctoral, Universitat Autònoma de Barcelona.

Ville-Benavides, R., 2015. Mal de montaña agudo en México: características clínicas y factores de riesgo. Tesis de especialidad de medicina interna. UNAM, http://132.248.9.195/ptd2017/agosto/514212421/ Index.html.

Vázquez-Selem, L., Heine, K., 2011. Chapter 61 - late quaternary glaciation in mexico. En: Ehlers, J., Gibbard, P. L., Hughes, P. D. (Eds.), Quaternary Glaciations - Extent and Chronology. Vol. 15 of Developments in Quaternary Sciences. Elsevier, pp. 849 - 861, doi:10.1016/B978-0-444-53447-7.000611 .

White, S. E., Reyes-Cortes, M., Ortega Ramírez, J., Valastro, S., 1990. El Ajusco : geomorfología volcánica y acontecimientos glaciales durante el Pleistoceno superior y comparación con las series glaciales mexicanas y las de las Montañas Rocallosas. Colección Científica, Serie Arqueología, INAH, México, D.F., 77 pp.

\section{This article accompanies the following material:}

HTML:

Static map:

Interactive map:

3D Interactive map:
DOI: 10.22201/igg.terradigitalis.2018.1.37.86

DOI: 10.22201/igg.terradigitalis.2018.1.37.99

DOI: 10.22201/igg.terradigitalis.2018.1.37.100

DOI: 10.22201/igg.terradigitalis.2018.1.37.101 


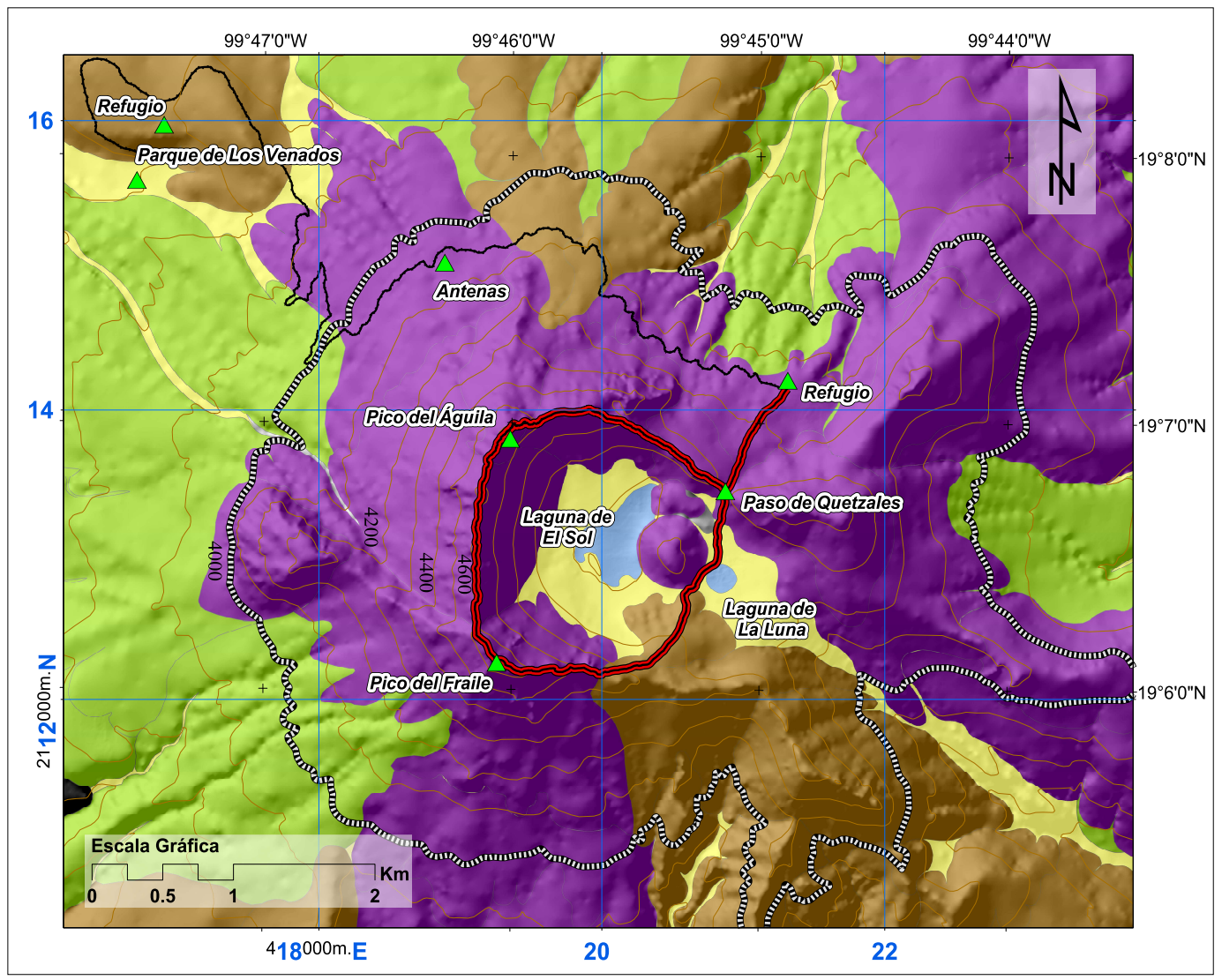

\begin{tabular}{|c|c|c|}
\hline \multicolumn{3}{|c|}{ Circunvalación Completa $\square$} \\
\hline Leyenda & \multicolumn{2}{|c|}{ Elementos geoturísticos } \\
\hline$\triangle$ Puntos de referencia & & Lagunas de El Sol y La Luna \\
\hline Circunvalación Completa & & Depósitos Piroclásticos \\
\hline mmmm Transición de bosque a pastizal alpino & & Nevado reciente $0.05 \mathrm{Ma}$-Presente \\
\hline Curva de nivel a cada $100 \mathrm{~m}$ & & Nevado viejo 1.5-0.13 Ma A.P. \\
\hline - Terracería de coches & & Depósitos aluviales y de glaciar \\
\hline \multicolumn{3}{|c|}{ Nivel de dificultad } \\
\hline & \multicolumn{2}{|c|}{ Muy Difícil } \\
\hline \multicolumn{3}{|c|}{$\begin{array}{l}\text { Recomendaciones } \\
\text { Equipo técnico, casco, arnés, botas, bastones, crampones, piolet y lentes obscuros. } \\
\text { Salud } \\
\text { Botiquín de primeros auxilios. Agua o electrolitos y comida energética. Estar atentos a } \\
\text { los síntomas del mal de montaña, ya que es una ruta que se pratica por arriba de los } \\
4000 \text { msnm. En caso de haber nieve, la dificultad de la ruta aumenta. }\end{array}$} \\
\hline \multicolumn{3}{|l|}{ Paisaje } \\
\hline \multicolumn{3}{|l|}{$\begin{array}{l}\text { Fauna } \\
\text { Invertebrados, aves, lagartijas. }\end{array}$} \\
\hline \multicolumn{3}{|l|}{$\begin{array}{l}\text { Tiempo de recorrido: } 9 \mathrm{hrs} \text {. } \\
\text { Altura máxima: } 4680 \mathrm{msnm}\end{array}$} \\
\hline $\begin{array}{l}\text { Distancia: } 8.5 \mathrm{~km} \text {. } \\
\square \text { Color que muestra la ruta en los mapas inte }\end{array}$ & ractivos $2 \mathrm{D}$ y $3 \mathrm{D}$ & 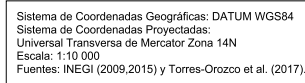 \\
\hline
\end{tabular}

Figura 1. Ruta Circunvalación Completa Figure 1. Circunvalación Completa path 


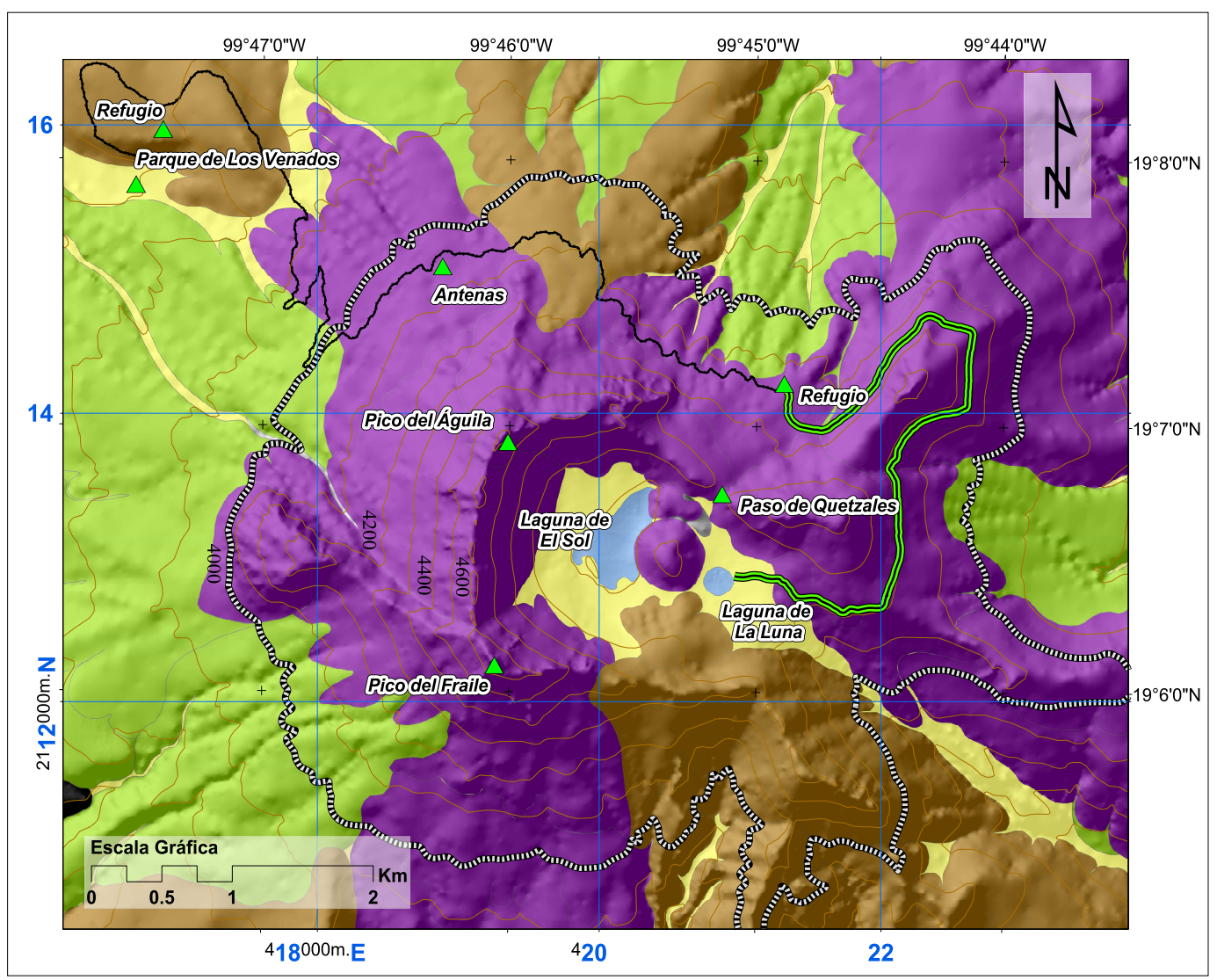

\section{Circunvalación Humboldt-Laguna de La Luna}

\section{Leyenda}

$\triangle$ Puntos de referencia

= Humboldt-Laguna de la Luna

mmmmm Transición de bosque a pastizal alpino Curva de nivel a cada $100 \mathrm{~m}$

—. Terracería de coches

\begin{tabular}{|l|l|l|l|}
\hline \multicolumn{4}{|c|}{ Nivel de dificultad } \\
\hline Fácil & & & \\
\hline
\end{tabular}

Recomendaciones

Equipo técnico, casco, arnés, botas, bastones, crampones, piolet y lentes obscuros.

\section{Salud}

Botiquín de primeros auxilios. Agua o electrolitos y comida energética. Estar atentos a los síntomas del mal de montaña, ya que es una ruta que se pratica por arriba de los $4000 \mathrm{msnm}$. En caso de haber nieve, la dificultad de la ruta aumenta.

Paisaje

Pastizal alpino, lagos del cráter.

Fauna

Invertebrados, aves, lagartijas.

Tiempo de recorrido: $4 \mathrm{hrs}$

Altura máxima: $4250 \mathrm{msnm}$

Distancia: $5.79 \mathrm{~km}$

$\square$ Color que muestra la ruta en los mapas interactivos $2 \mathrm{D}$ y $3 \mathrm{D}$ 


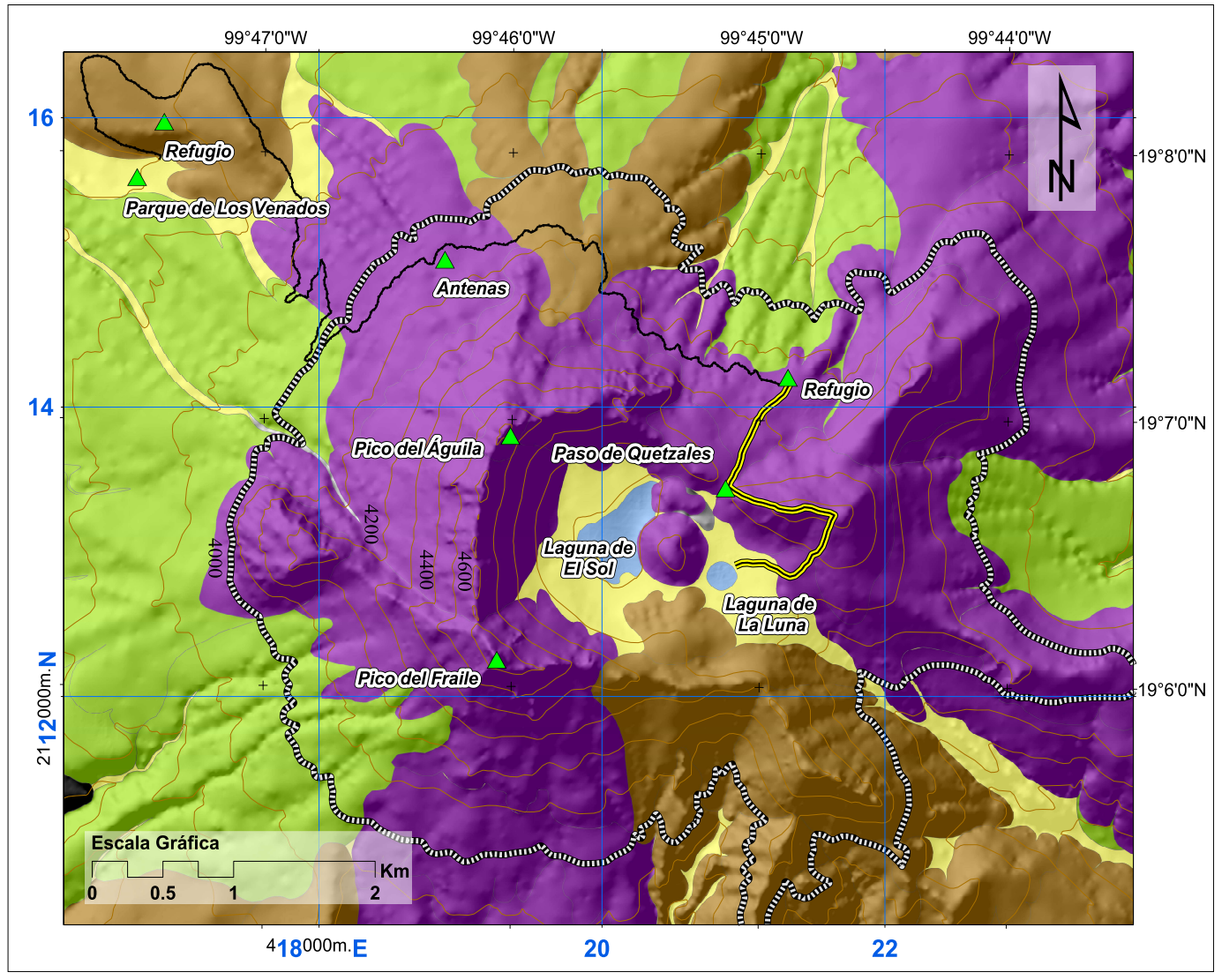

Figura 3. Ruta Humboldt Figure 3. Humboldt path

\section{Humboldt}

\section{Leyenda}

$\triangle$ Puntos de referencia

Humboldt

mmmm Transición de bosque a pastizal alpino Curva de nivel a cada $100 \mathrm{~m}$

- Terracería de coches

\begin{tabular}{|l|l|l|l|}
\hline \hline \multicolumn{4}{|c|}{ Nivel de dificultad } \\
\hline & Algo Difícil & & \\
\hline
\end{tabular}

\section{Recomendaciones}

Equipo técnico, casco, botas, bastones y lentes obscuros.

Salud

Agua o electrolitos y comida energética. Estar atentos a los síntomas del mal de

montaña, ya que es una ruta que se practica por arriba de los $4000 \mathrm{msnm}$

En caso de haber nieve, la dificultad de la ruta aumenta.

\section{Paisaje}

Pastizal alpino, rosa de montaña, lagos del cráter.

\section{Fauna}

Invertebrados, aves, lagartijas.

Tiempo de recorrido: $2 \mathrm{hrs}$

Altura máxima: $4450 \mathrm{msnm}$

Distancia: $2.78 \mathrm{~km}$.

$\square$ Color que muestra la ruta en los mapas interactivos $2 \mathrm{D}$ y $3 \mathrm{D}$

\section{Elementos geoturísticos}

Lagunas de El Sol y La Luna

Depósitos Piroclásticos

Nevado reciente $0.05 \mathrm{Ma}$-Presente Nevado viejo 1.5-0.13 Ma A.P. Depósitos aluviales y de glaciar

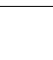




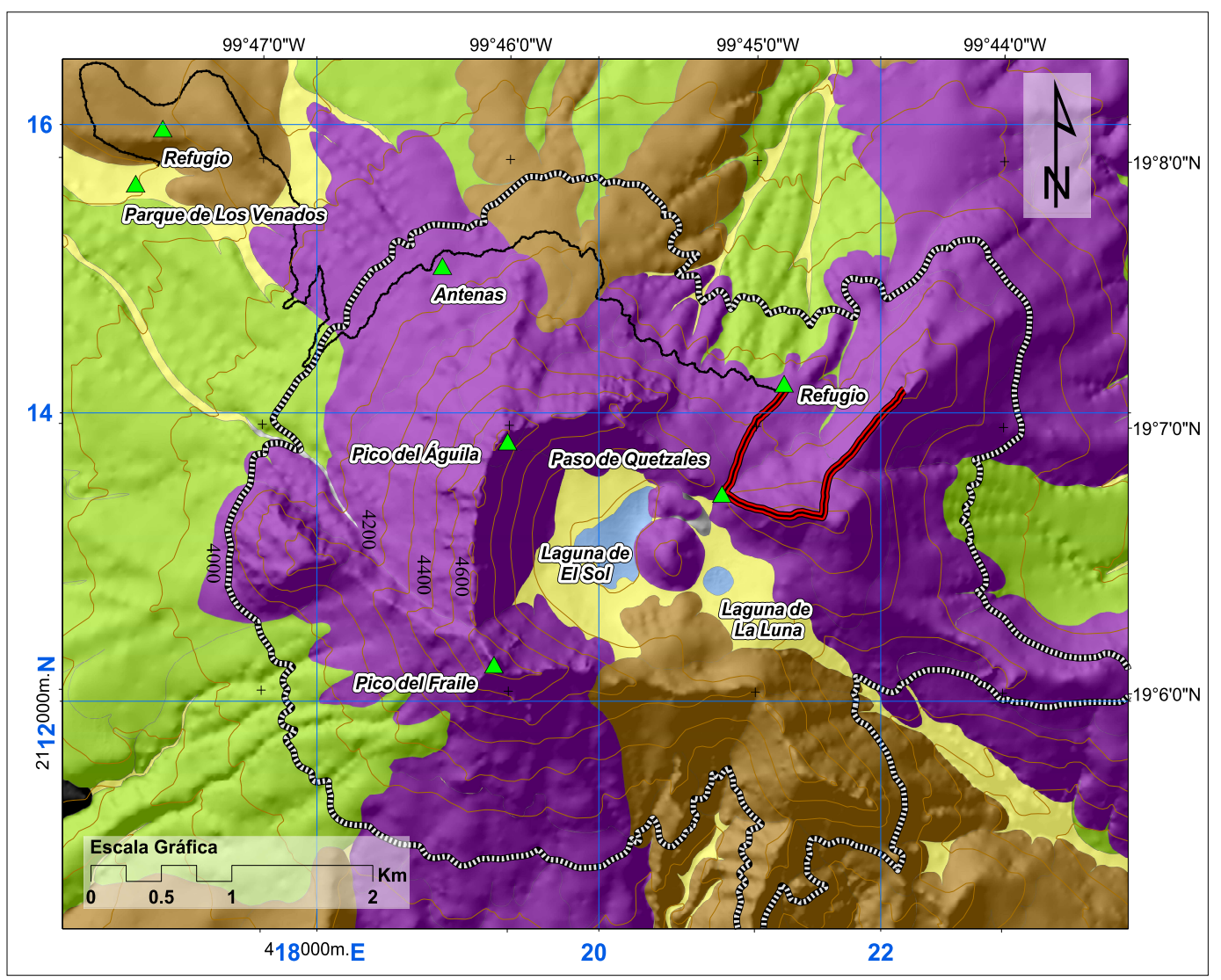

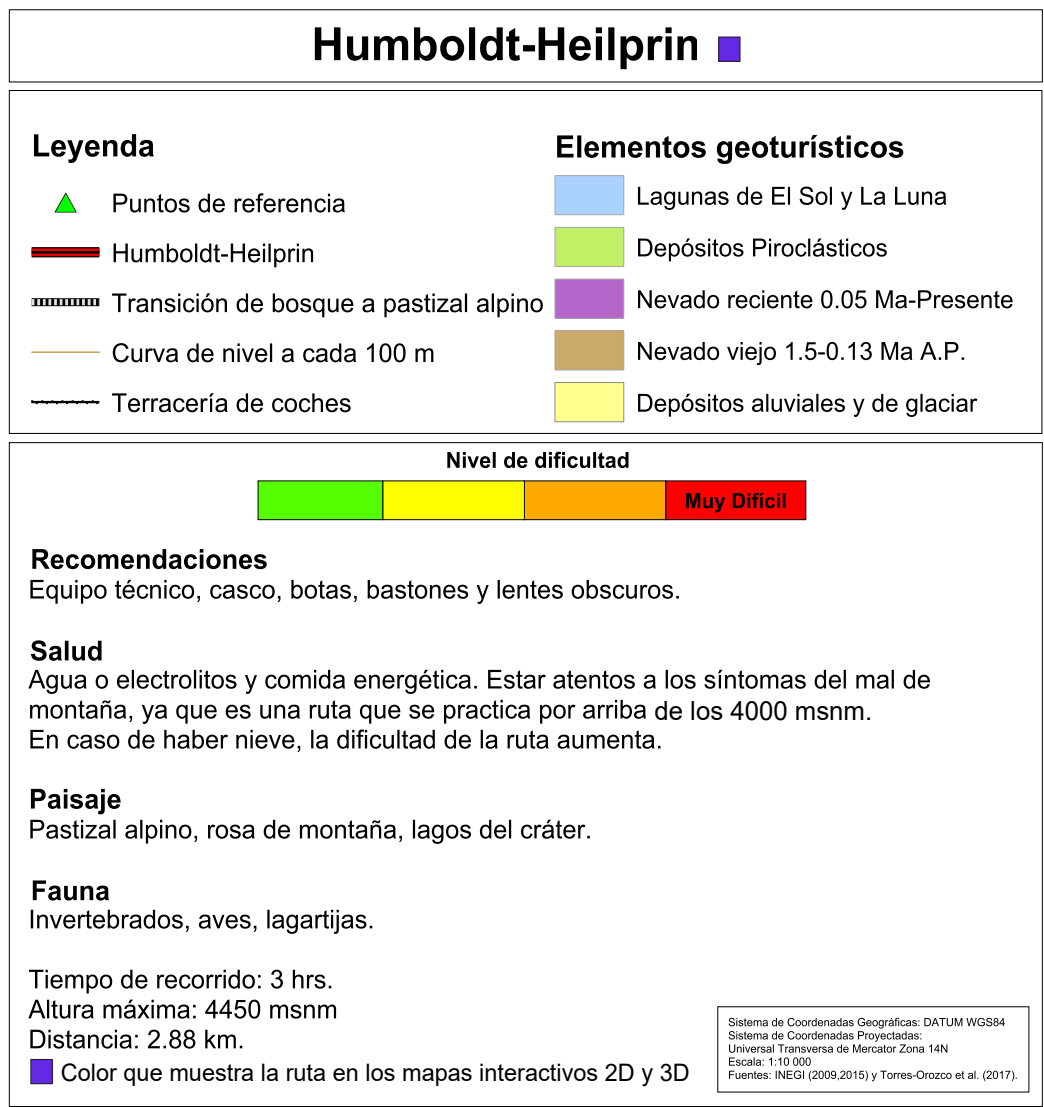

Figura 4. Ruta Humboldt-Heilprin Figure 4. Humboldt-Heilprin path 


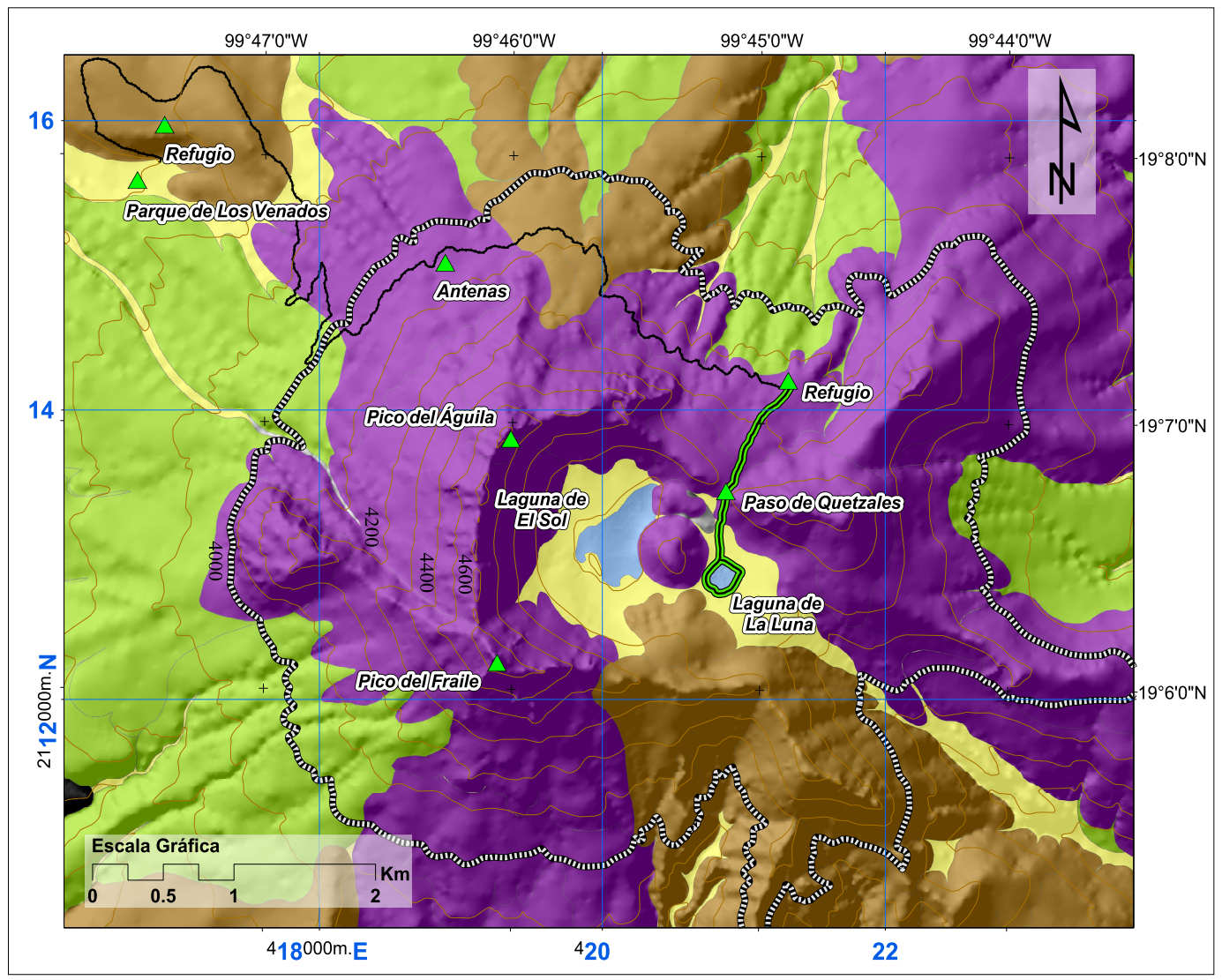

\begin{tabular}{|c|c|c|}
\hline \multicolumn{3}{|c|}{ Laguna de La Luna $\square$} \\
\hline Leyenda & \multicolumn{2}{|c|}{ Elementos geoturísticos } \\
\hline$\triangle \quad$ Puntos de referencia & & Lagunas de El Sol y La Luna \\
\hline$\overline{\overline{\underline{ }}}$ Laguna de La Luna & & Depósitos Piroclásticos \\
\hline \multirow{3}{*}{$\begin{array}{l}\text { mmmm Transición de bosque a pastizal alpino } \\
\text { Curva de nivel a cada } 100 \mathrm{~m} \\
\text { _ Terracería de coches }\end{array}$} & & Nevado reciente $0.05 \mathrm{Ma}$-Presente \\
\hline & & Nevado viejo $1.5-0.13$ Ma A.P. \\
\hline & & Depósitos aluviales y de glaciar \\
\hline \multicolumn{3}{|c|}{ Nivel de dificultad } \\
\hline \multicolumn{3}{|c|}{ 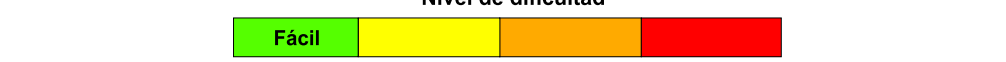 } \\
\hline \multicolumn{3}{|c|}{$\begin{array}{l}\text { Recomendaciones } \\
\text { Ropa abrigadora, botas, bastones y lentes obscuros. }\end{array}$} \\
\hline \multicolumn{3}{|c|}{$\begin{array}{l}\text { Salud } \\
\text { Agua o electrolitos y comida energética. Estar atentos a los síntomas del mal de } \\
\text { montaña, ya que es una ruta que se practica por arriba de los } 4000 \mathrm{msnm} \text {. En caso } \\
\text { de haber nieve, la dificultad de la ruta aumenta. }\end{array}$} \\
\hline \multicolumn{3}{|l|}{ Paisaje } \\
\hline \multicolumn{3}{|l|}{$\begin{array}{l}\text { Fauna } \\
\text { Invertebrados, aves, lagartijas. }\end{array}$} \\
\hline \multicolumn{2}{|c|}{$\begin{array}{l}\text { Tiempo de recorrido: } 2 \mathrm{hrs} \\
\text { Altura máxima: } 4300 \mathrm{msnm} \\
\text { Distancia: } 4.23 \mathrm{~km} \text {. } \\
\square \text { Color que muestra la ruta en los mapas interactivos } 2 \mathrm{D} \text { y } 3 \mathrm{D}\end{array}$} & 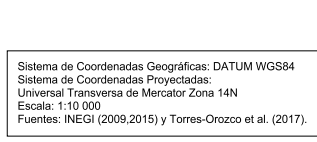 \\
\hline
\end{tabular}

Figura 5. Ruta Laguna de La Luna Figure 5. Laguna de La Luna path 


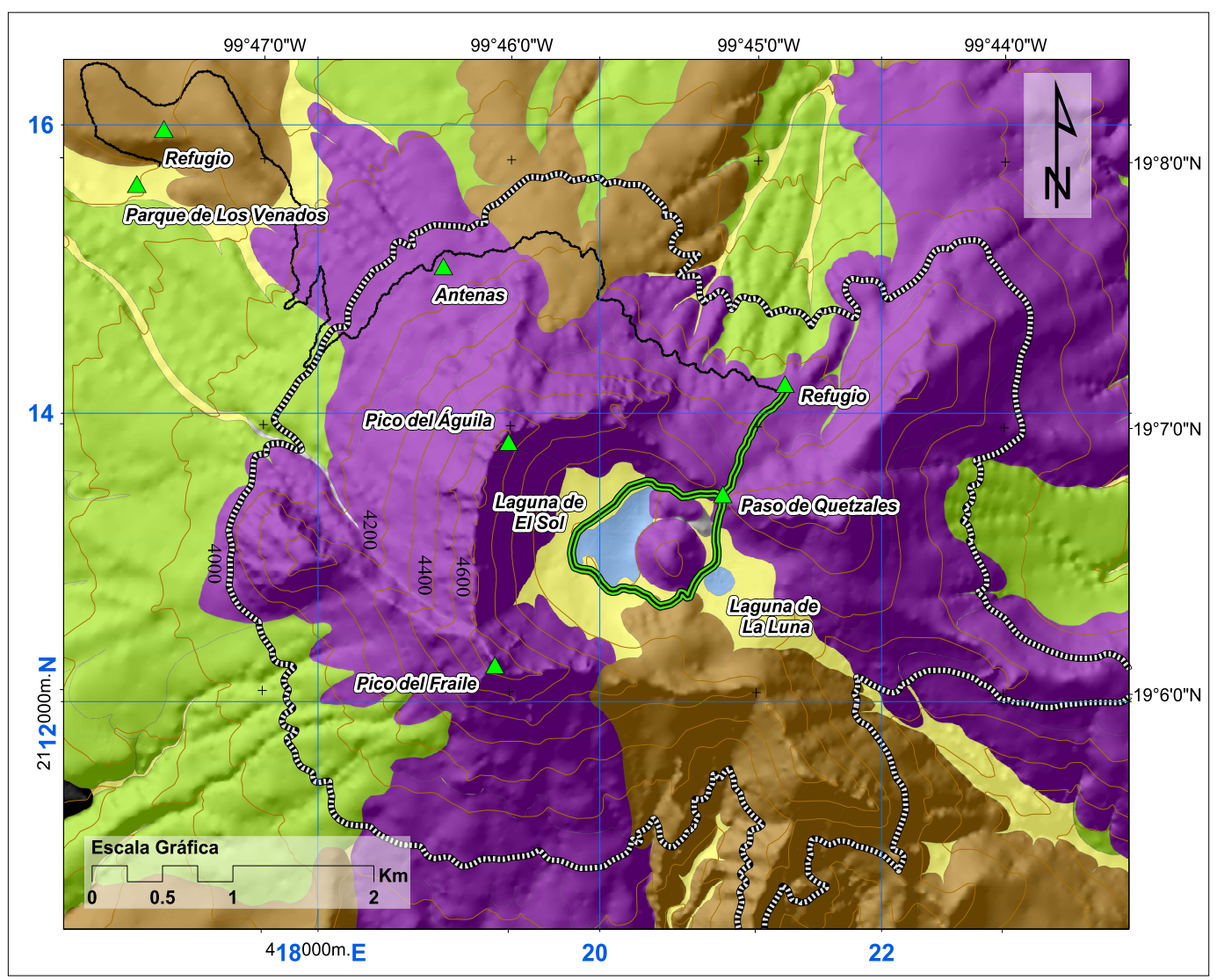

\begin{tabular}{|c|c|c|}
\hline \multicolumn{3}{|c|}{ Laguna del Sol $\square$} \\
\hline Leyenda & \multicolumn{2}{|c|}{ Elementos geoturísticos } \\
\hline$\triangle \quad$ Puntos de referencia & & Lagunas de El Sol y La Luna \\
\hline$\overline{\overline{\underline{ }}}$ Laguna del Sol & & Depósitos Piroclásticos \\
\hline \multirow{3}{*}{$\begin{array}{l}\text { mmmm Transición de bosque a pastizal alpino } \\
\text { Curva de nivel a cada } 100 \mathrm{~m} \\
\ldots \text { Terracería de coches }\end{array}$} & & Nevado reciente 0.05 Ma-Presente \\
\hline & & Nevado viejo 1.5-0.13 Ma A.P. \\
\hline & & Depósitos aluviales y de glaciar \\
\hline \multicolumn{3}{|c|}{ Nivel de dificultad } \\
\hline \multicolumn{3}{|l|}{ Fácil } \\
\hline \multicolumn{3}{|c|}{$\begin{array}{l}\text { Recomendaciones } \\
\text { Ropa abrigadora, botas, bastones y lentes obscuros. }\end{array}$} \\
\hline \multicolumn{3}{|c|}{$\begin{array}{l}\text { Salud } \\
\text { Agua o electrolitos y comida energética. Estar atentos a los síntomas del mal de } \\
\text { montaña, ya que es una ruta que se practica por arriba de los } 4000 \mathrm{msnm} \text {. } \\
\text { En caso de haber nieve, la dificultad de la ruta aumenta. }\end{array}$} \\
\hline \multicolumn{3}{|l|}{$\begin{array}{l}\text { Paisaje } \\
\text { Pastizal alpino, lagos del cráter. }\end{array}$} \\
\hline \multicolumn{3}{|l|}{$\begin{array}{l}\text { Fauna } \\
\text { Invertebrados, aves, lagartijas. }\end{array}$} \\
\hline \multicolumn{3}{|l|}{ Tiempo de recorrido: $3 \mathrm{hrs}$. } \\
\hline $\begin{array}{l}\text { Distancia: } 7.44 \mathrm{~km} \text {. } \\
\square \text { Color que muestra la ruta en los mapas intera }\end{array}$ & ctivos $2 \mathrm{D}$ y $3 \mathrm{D}$ & 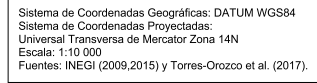 \\
\hline
\end{tabular}

Figura 6. Ruta Laguna del So Figure 6. Laguna del Sol path 


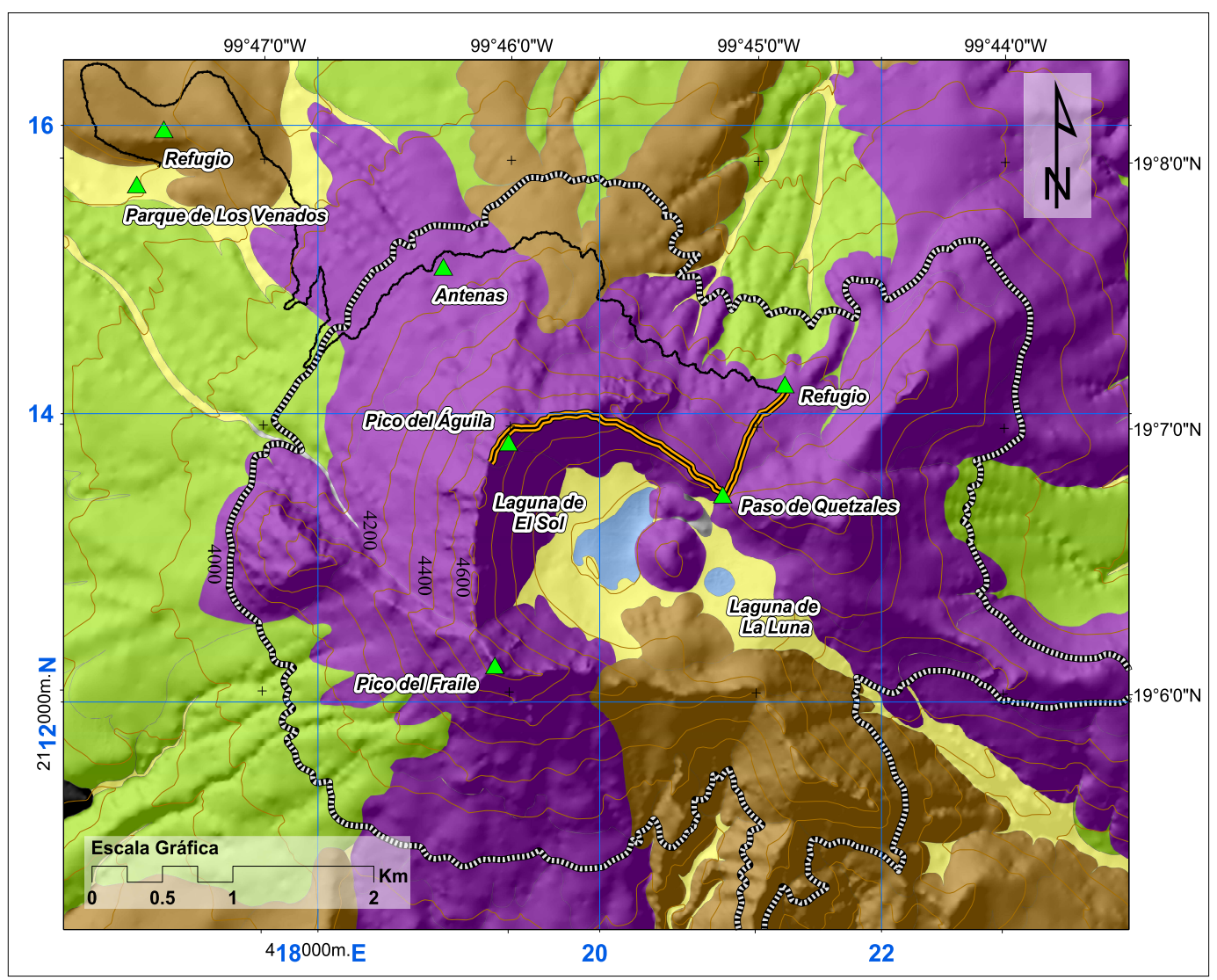

\begin{tabular}{|c|c|c|c|}
\hline \multicolumn{4}{|c|}{ Pico del Águila $\square$} \\
\hline \multicolumn{2}{|c|}{ Leyenda } & \multicolumn{2}{|c|}{ Elementos geoturísticos } \\
\hline$\triangle$ & Puntos de referencia & & Lagunas de El Sol y La Luna \\
\hline & Pico del Águila & & Depósitos Piroclásticos \\
\hline \multirow[t]{3}{*}{ mmmmm } & \multirow{3}{*}{$\begin{array}{l}\text { Transición de bosque a pastizal alpino } \\
\text { Curva de nivel a cada } 100 \mathrm{~m} \\
\text { Terracería de coches }\end{array}$} & & Nevado reciente $0.05 \mathrm{Ma}$-Presente \\
\hline & & & Nevado viejo $1.5-0.13$ Ma A.P. \\
\hline & & & Depósitos aluviales y de glaciar \\
\hline \multicolumn{4}{|c|}{ Nivel de dificultad } \\
\hline & & Díficil & \\
\hline
\end{tabular}

\section{Recomendaciones}

Equipo técnico, casco, arnés, botas, bastones, crampones, piolet y lentes obscuros.

Salud

Botiquin de primeros auxilios. Agua o electrolitos y comida energética. Estar atentos a los síntomas del mal de montaña, ya que es una ruta que se practica por arriba de los $4000 \mathrm{msnm}$. En caso de haber nieve, la dificultad de la ruta aumenta.

Paisaje

Pastizal alpino, lagos del cráter.

Fauna

Invertebrados, aves, lagartijas.

Tiempo de recorrido: $5 \mathrm{hrs}$

Altura máxima: $4660 \mathrm{msnm}$

Distancia: $2.83 \mathrm{~km}$.

$\square$ Color que muestra la ruta en los mapas interactivos $2 \mathrm{D}$ y $3 \mathrm{D}$

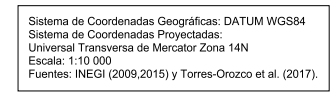

Figura 7. Ruta Pico del Águila Figure 7. Pico del Águila path 


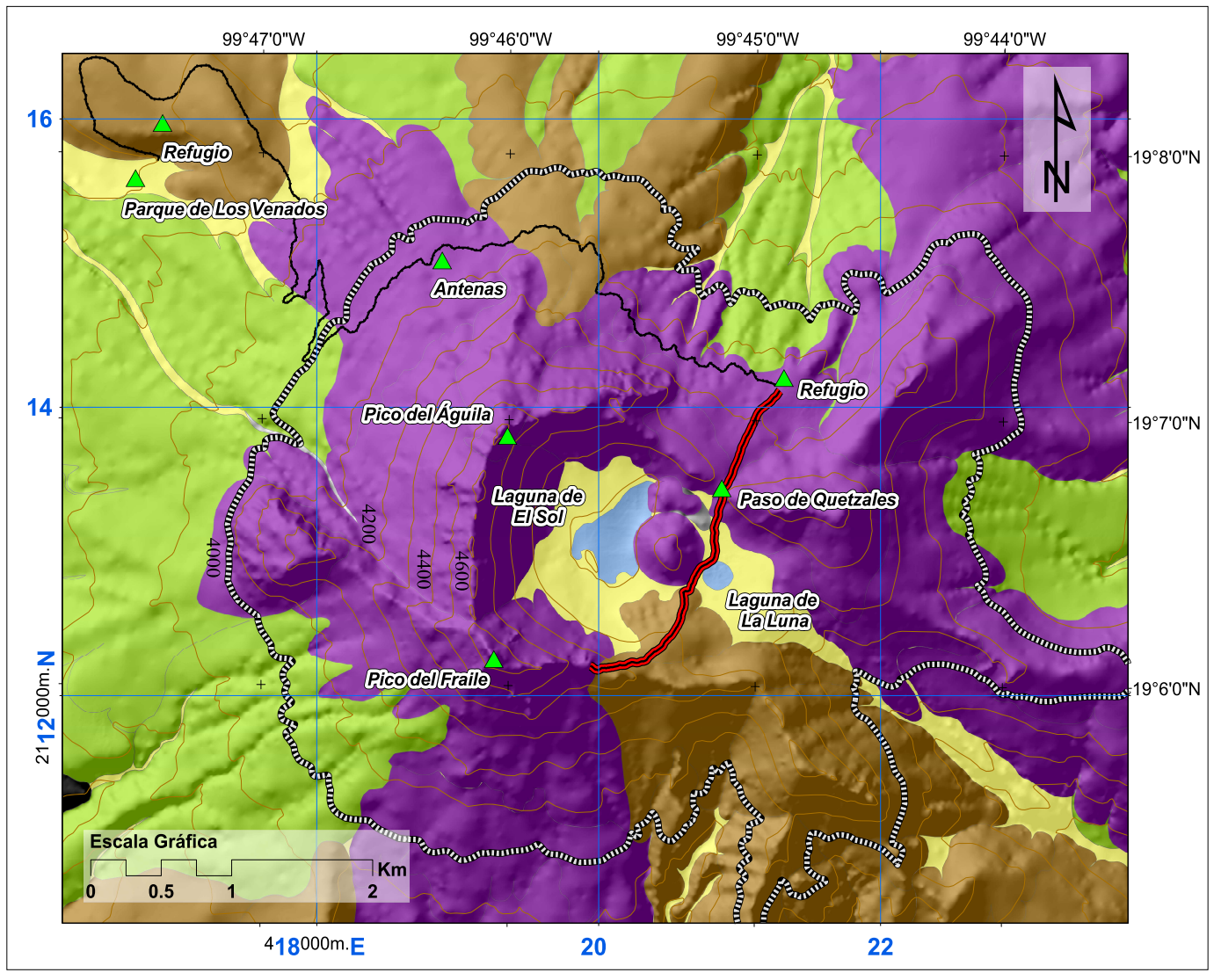

Figura 8. Ruta Pico del Fraile Figure 8. Pico del Fraile path

\section{Pico del Fraile}

\section{Leyenda}

$\triangle$ Puntos de referencia

Pico del Fraile

mmmmm Transición de bosque a pastizal alpino Curva de nivel a cada $100 \mathrm{~m}$

- Terracería de coches

\begin{tabular}{|l|l|l|l|}
\hline \multicolumn{4}{|c|}{ Nivel de dificultad } \\
$\qquad$ & & & Muy Dificil \\
\hline
\end{tabular}

\section{Recomendaciones}

Equipo técnico, casco, arnés, botas, bastones, crampones, piolet y lentes obscuros.

Salud

Botiquin de primeros auxilios. Agua o electrolitos y comida energética. Estar atentos a los síntomas del mal de montaña, ya que es una ruta que se practica por arriba de los $4000 \mathrm{msnm}$. En caso de haber nieve, la dificultad de la ruta aumenta.

Paisaje

Pastizal alpino, lagos del cráter.

Fauna

Invertebrados, aves, lagartijas.

Tiempo de recorrido: $7 \mathrm{hrs}$

Altura máxima: $4680 \mathrm{msnm}$

Distancia: $3.09 \mathrm{~km}$.

$\square$ Color que muestra la ruta en los mapas interactivos $2 \mathrm{D}$ y $3 \mathrm{D}$

\section{Elementos geoturísticos}

Lagunas de El Sol y La Luna

Depósitos Piroclásticos

Nevado reciente 0.05 Ma-Presente

Nevado viejo 1.5-0.13 Ma A.P.

Devado viejo $1.5-0.13$

Depósitos aluviales y de glaciar 


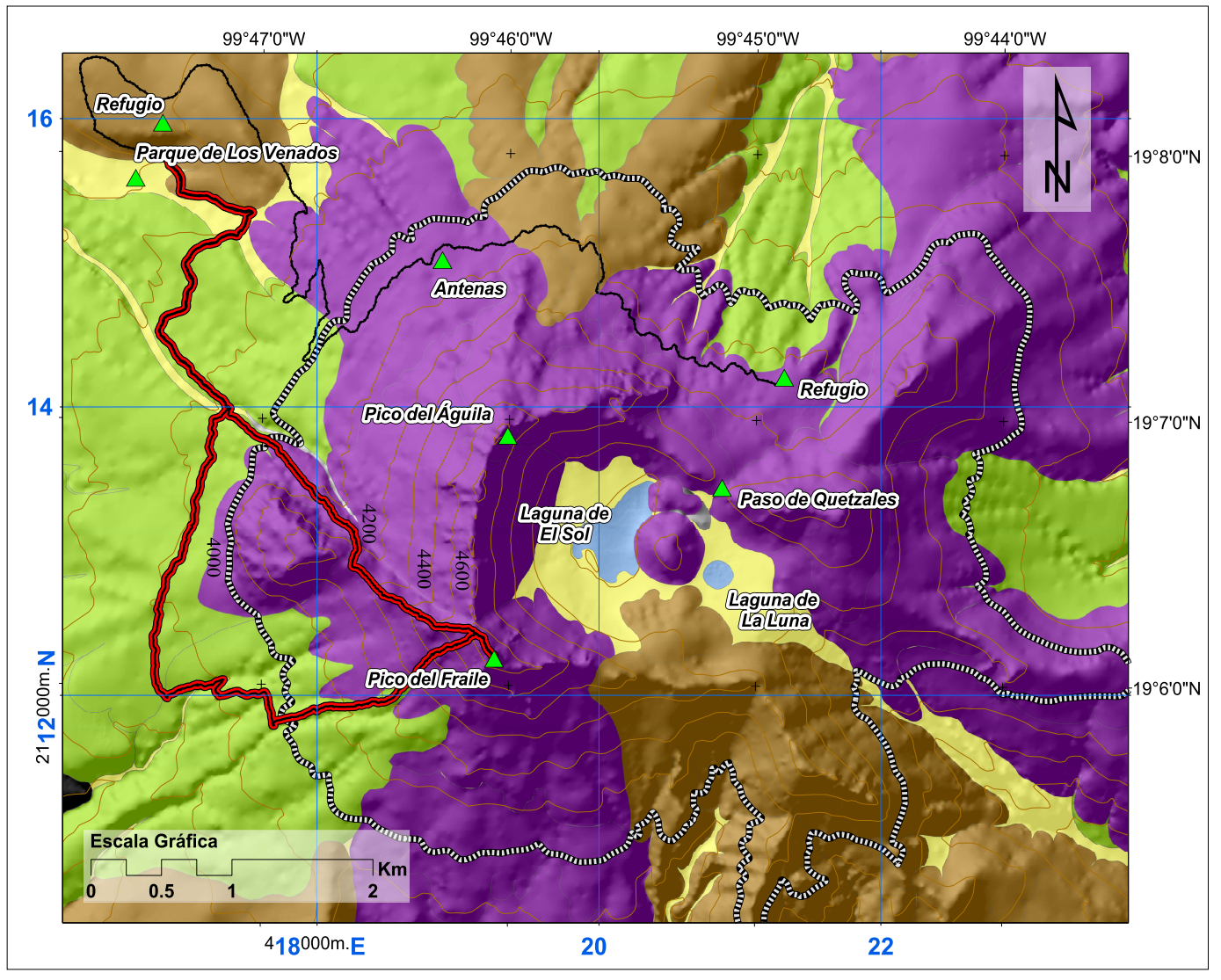

\section{Parque de Los Venados-Cara Sur}

\section{Leyenda}

$\triangle$ Puntos de referencia

\section{Venados-cara sur}

mmmm Transición de bosque a pastizal alpino

Curva de nivel a cada $100 \mathrm{~m}$

- Terracería de coches

\begin{tabular}{|l|l|l|l|}
\hline \multicolumn{4}{|c|}{ Nivel de dificultad } \\
\cline { 2 - 4 } & & & Muy Dificil \\
\hline
\end{tabular}

\section{Recomendaciones}

Equipo técnico, casco, arnés, botas, bastones, crampones, piolet y lentes obscuros.

\section{Salud}

Botiquín de primeros auxilios. Agua o electrolitos y comida energética. Estar atentos a los sintomas del mal de montaña, ya que es una ruta que se practica por arriba de los $4000 \mathrm{msnm}$. En caso de haber nieve, la dificultad de la ruta aumenta.

\section{Paisaje}

Bosque de coníferas con especies dominantes a medida que se asciende: oyamel y ocote, pastizal alpino, lagos del cráter.

Fauna

Invertebrados, aves, lagartijas, tuza, coyote y venado.

Tiempo de recorrido: $8 \mathrm{hrs}$

Altura máxima: $4600 \mathrm{msnm}$

Distancia: $14.4 \mathrm{~km}$.

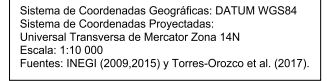

Figura 9. Ruta Parque de Los Venados-Cara Su Figure 9. Parque de Los Venados-Cara Sur path 


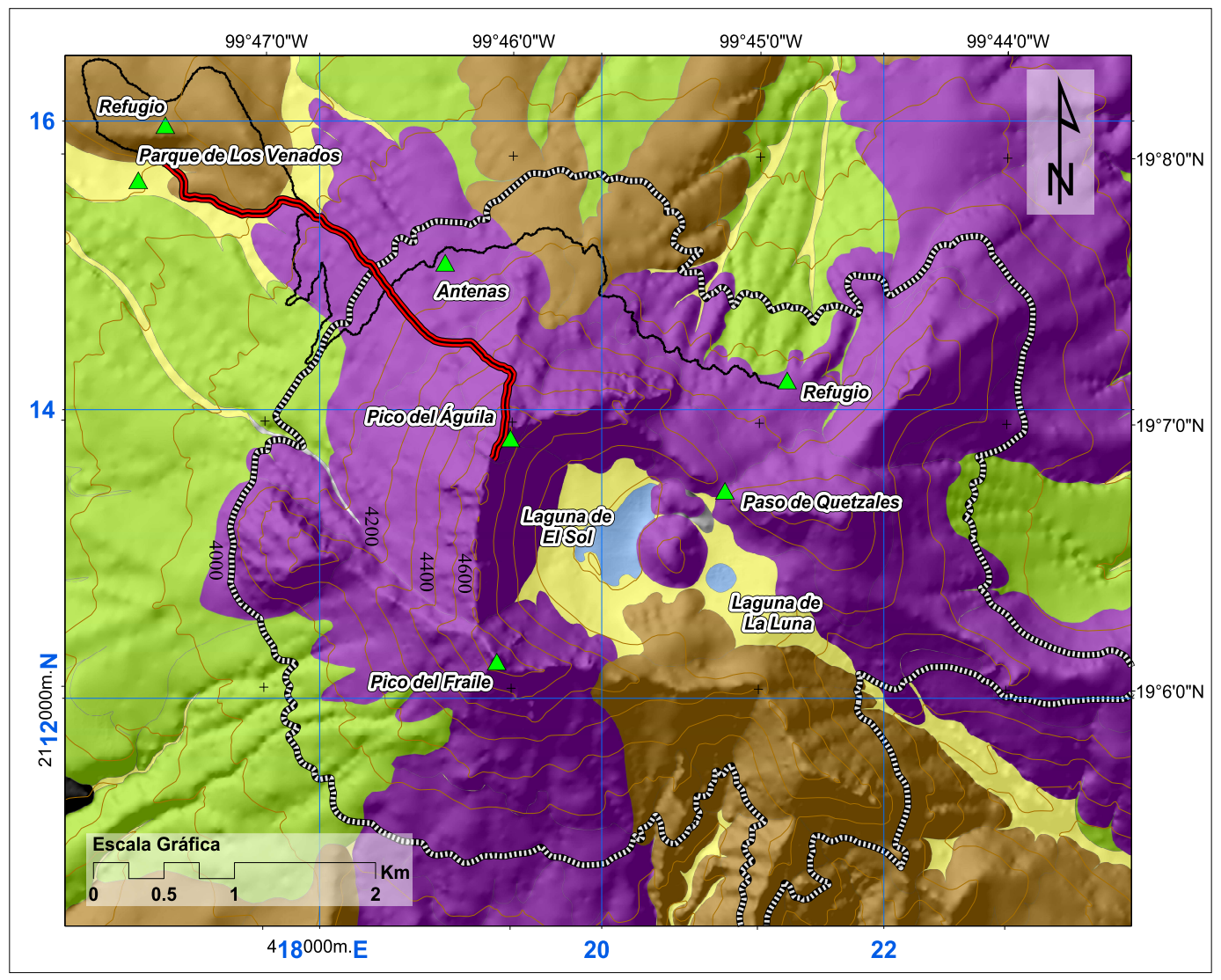

\section{Parque de Los Venados-Pico del Águila}

\section{Leyenda}

$\triangle$ Puntos de referencia

P. de los Venados-Pico del Águila

mmmIm Transición de bosque a pastizal alpino

Curva de nivel a cada $100 \mathrm{~m}$

- Terracería de coches

\begin{tabular}{|l|l|l|l|}
\hline \multicolumn{3}{|c|}{ Nivel de dificultad } \\
$\qquad$ & & & Muy Dificil \\
\hline
\end{tabular}

\section{Recomendaciones}

Equipo técnico, casco, arnés, botas, bastones, crampones, piolet y lentes obscuros.

\section{Salud}

Botiquín de primeros auxilios. Agua o electrolitos y comida energética. Estar atentos a los síntomas del mal de montaña, ya que es una ruta que se practica por arriba de los $4000 \mathrm{msnm}$. En caso de haber nieve, la dificultad de la ruta aumenta.

\section{Paisaje}

Bosque de coníferas con especies dominantes a medida que se asciende: oyamel y ocote, pastizal alpino, lagos del cráter.

Fauna

Invertebrados, aves, lagartijas, tuza, coyote y venado.

Tiempo de recorrido: $8 \mathrm{hrs}$

Altura máxima: $4640 \mathrm{msnm}$

Distancia: $4.09 \mathrm{~km}$.

Color que muestra la ruta en los mapas interactivos $2 \mathrm{D}$ y $3 \mathrm{D}$ 


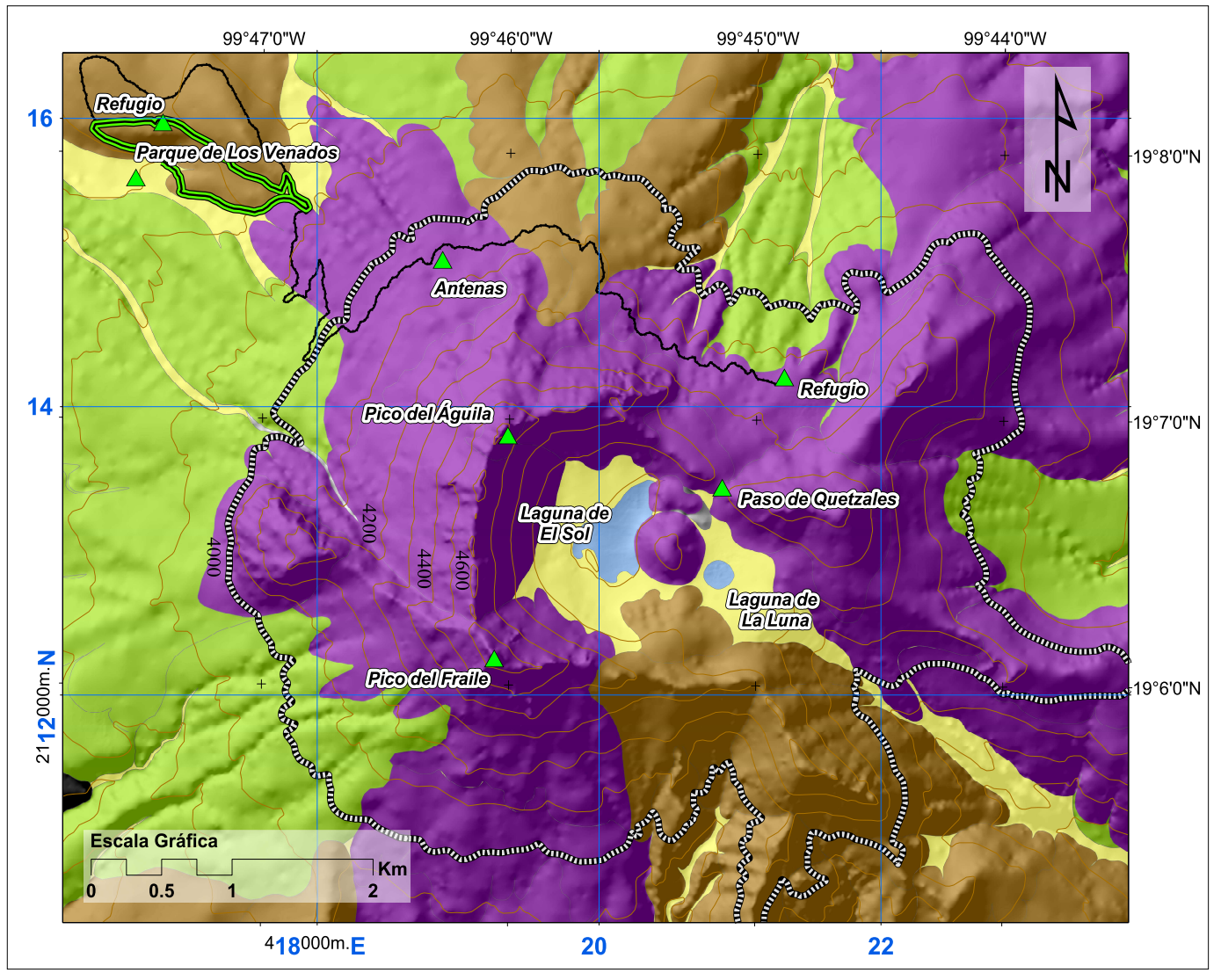

Figura 11. Sendero Interpretativo Figure 11. Interpretative path

\section{Sendero Interpretativo}

\section{Leyenda}

$\triangle$ Puntos de referencia

= sendero interpretativo

mmmmm Transición de bosque a pastizal alpin。

_ Curva de nivel a cada $100 \mathrm{~m}$

- Terracería de coches

\begin{tabular}{|l|l|l|l|}
\hline \multicolumn{4}{|c|}{ Nivel de dificultad } \\
\hline Fácil & & & \\
\hline
\end{tabular}

Recomendaciones

Ropa abrigadora, impermeable, botas y lentes obscuros.

\section{Salud}

Agua o electrolitos y comida energética. En caso de haber nieve, la dificultad de la ruta aumenta.

\section{Paisaje}

Bosque de coníferas con especies dominantes a medida que se asciende: oyamel y ocote, pastizal alpino, lagos del cráter.

Fauna

Invertebrados, aves, lagartijas, tuza, coyote y venado.

Tiempo de recorrido: $3 \mathrm{hrs}$.

Altura máxima: $4000 \mathrm{msnm}$

Distancia: $4 \mathrm{~km}$.

Color que muestra la ruta en los mapas interactivos $2 \mathrm{D}$ y $3 \mathrm{D}$

\section{Eementurísticos}

Lagunas de El Sol y La Luna

Depósitos Piroclásticos

Nevado reciente $0.05 \mathrm{Ma}$-Presente

Nevado viejo 1.5-0.13 Ma A.P.

Depósitos aluviales y de glacia 


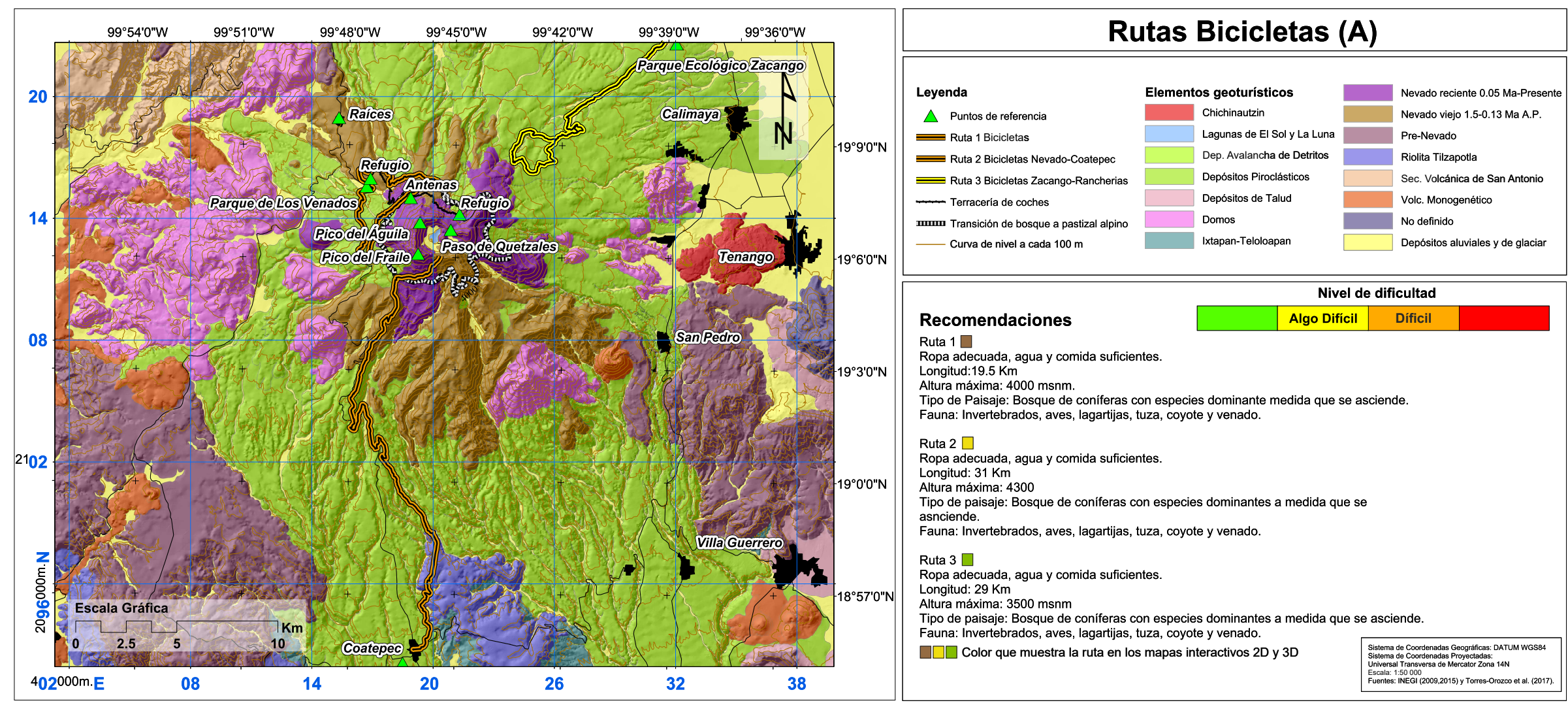

Figura 12. Rutas Bicicletas (A)

Figure 12. Bicycle paths A 


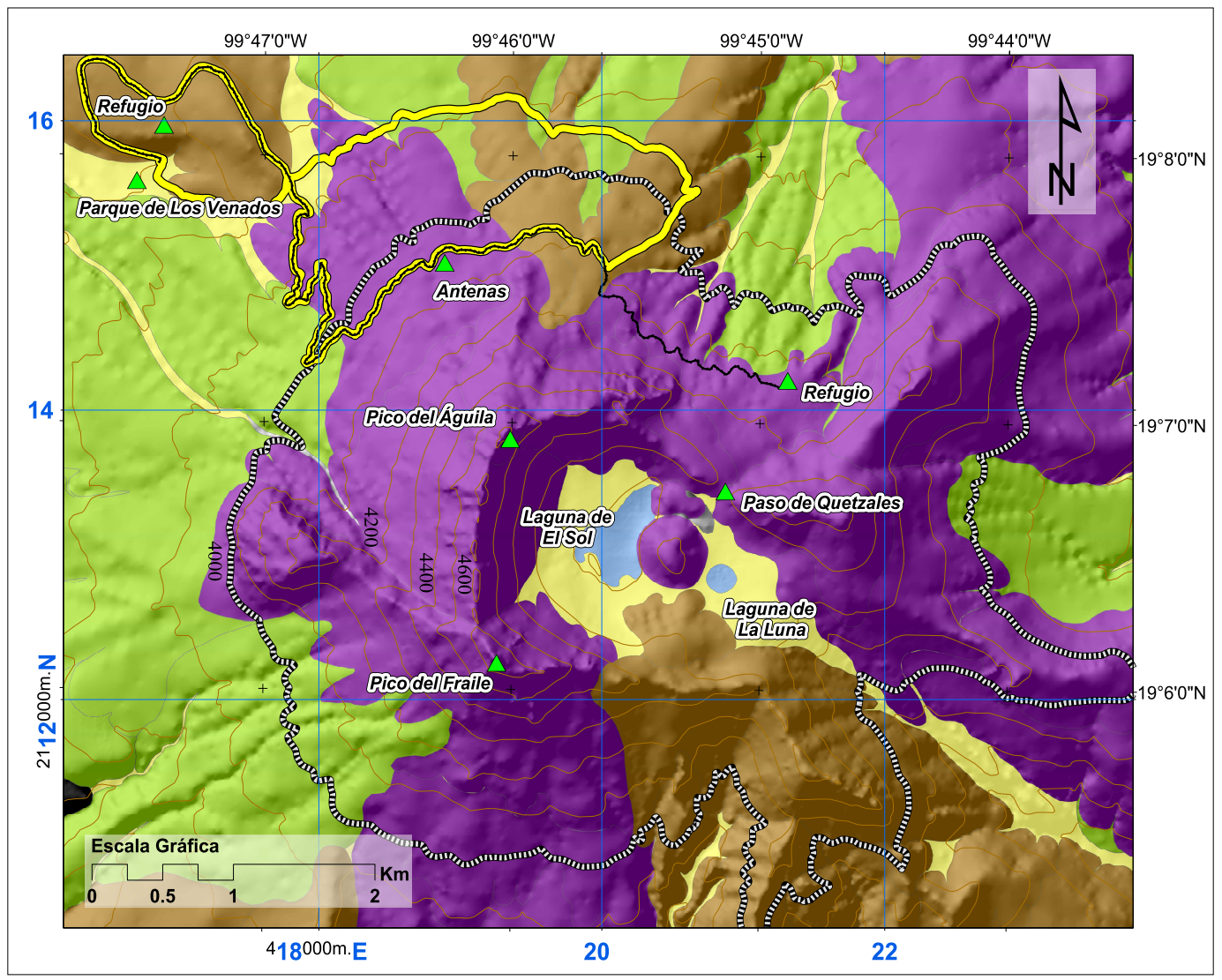

\begin{tabular}{|c|c|c|}
\hline \multicolumn{3}{|c|}{ Bicicletas (B) } \\
\hline Leyenda & \multicolumn{2}{|c|}{ Elementos geoturísticos } \\
\hline$\triangle$ Puntos de referencia & & Lagunas de El Sol y La Luna \\
\hline$\overline{\overline{ }}$ Ruta 4. Dunas & & Depósitos Piroclásticos \\
\hline Luta $5^{*}$. Terracería & & Nevado reciente $0.05 \mathrm{Ma}$-Presente \\
\hline \multirow{2}{*}{$\begin{array}{l}\text { mmmm Transición de bosque a pastizal alpino } \\
\text { Curva de nivel a cada } 100 \mathrm{~m}\end{array}$} & & Nevado viejo 1.5-0.13 Ma A.P. \\
\hline & & Depósitos aluviales y de glaciar \\
\hline \multicolumn{3}{|c|}{ Nivel de dificultad } \\
\hline Algo Difícil & & \\
\hline \multicolumn{3}{|c|}{$\begin{array}{l}\text { Recomendaciones } \\
\text { Ruta } 4 \square \\
\text { Ropa adecuada, agua y comida suficientes. } \\
\text { Longitud: } 18.7 \mathrm{Km} \\
\text { Altura máxima: } 4150 \mathrm{msnm} \text {. } \\
\text { Tipo de Paisaje: Bosque de ocote. } \\
\text { Fauna: Invertebrados, aves, lagartijas, tuza, coyote y venado. }\end{array}$} \\
\hline $\begin{array}{l}\text { Ruta } 5 \\
\text { Ropa adecuada, agua y comida suficientes } \\
\text { Longitud: } 10.6 \mathrm{Km} \\
\text { Altura máxima: } 4300 \\
\text { Tipo de paisaje: Bosque de pino de las altu } \\
\text { Fauna: Invertebrados, aves, lagartijas, tuza }\end{array}$ & $\begin{array}{l}\text { s. } \\
\text { uras y pastizal alpi } \\
\text { a, coyote y venado }\end{array}$ & \\
\hline \multicolumn{2}{|c|}{$\begin{array}{l}{ }^{*} \text { El camino de terrecaría que conduce al refugio } \\
\text { de quetzales. Nivel de dificultad: Difícil } \\
\square \square \text { Color que muestra la ruta en los mapas interactivos } 2 \mathrm{D} \text { y } 3 \mathrm{D}\end{array}$} & 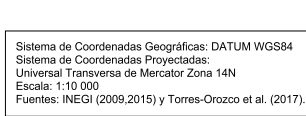 \\
\hline
\end{tabular}

Figura 13. Rutas Bicicletas (B) Figure 13. Bicycle paths (B) 